\title{
Análisis comparativo de un cambio fonológico erosivo. Variación de /d/ intervocálica en dos comunidades de habla (Granada y Málaga)
}

\author{
Juan Andrés Villena Ponsoda* \\ Universidad de Málaga \\ Juan Antonio Moya Corral ${ }^{* *}$ \\ Universidad de Granada
}

\begin{abstract}
Resumen
El objetivo de este trabajo es el desarrollo de un análisis sociolingüístico comparativo de la variación de la obstruyente /d/ en posición intervocálica en las variedades urbanas de Granada y Málaga (España), a partir de dos corpus orales diseñados y recolectados en el marco del Proyecto Preseea y, por tanto, perfectamente homologables. Dada la frecuencia de la elisión de esta variable en el español meridional de España, el análisis se redujo a la alternancia de mantenimiento y pérdida del segmento consonántico, obviándose diferencias de intensidad o tensión en las variantes consonánticas conservadas. Para ello se llevó a cabo un análisis bivariante exhaustivo previo tomando en consideración variables independientes lingüísticas y de hablante (estilísticas y sociales) y su interacción respectiva. A continuación, se desarrollaron dos análisis comparativos multivariantes, uno paralelo
\end{abstract}

Para correspondencia, dirigirse a: Juan Andrés Villena Ponsoda (vum@uma.es), Departamento de Filología Griega, Estudios Árabes, Lingüística General y Documentación, Universidad de Málaga, Facultad de Filosofía y Letras, Campus Universitario de Teatinos, s/n, 29071 Málaga, España.

** Para correspondencia, dirigirse a: Juan Antonio Moya Corral (jmoya@ugr.es), Departamento de Lengua Española, Facultad de Filosofía y Letras, Campus Universitario de Cartuja, s/n, Universidad de Granada 18071 Granada, España. 
de los datos de cada corpus y otro integrado de todos los datos. Los resultados - que son sorprendentemente similares en ambas ciudadesconfirman los obtenidos en otras áreas españolas y americanas en el sentido del peso fundamental de los factores internos (retención del segmento consonántico en la raíz y elisión en los morfemas) pero entran en contradicción parcialmente con respecto al efecto de los factores de hablante (avance de la elisión entre los jóvenes). Los ámbitos de conflicto indican una estratificación social y etaria más fuerte en Málaga que en Granada.

Palabras clave: sociolingüística, variación lingüística, análisis cuantitativo, variedades del español de Andalucía.

\title{
VARIATION OF INTERVOCALIC /d/ IN TWO SPEECH COMMUNITIES (Granada and Malaga). A comparative analysis of an EROSIVE PHONOLOGICAL CHANGE
}

\begin{abstract}
The aim of this paper is to carry out a comparative sociolinguistic analysis of intervocalic obstruent consonant /d/ variation in urban Granada and Malaga (Spain). To do so, two perfectly comparable spoken corpuses gathered in the frame of the PRESEEA-Project were used. Given that $/ \mathrm{d} /$ deletion is quite frequent in southern varieties of Castilian Spanish, our analysis zoomed in on consonant deletion vs. maintenance regardless of the differences of intensity or tension perceptible in maintained consonantal variants. Prior to the comparative analysis itself, an exhaustive bivariate analysis was performed considering both linguistic and speaker (stylistic and social) variables, as well as their respective interaction. Therefore, we carried out two multivariate comparative analyses, the first one involving separate parallel analyses of data from both corpuses and the second one being an integrated analysis of the whole data. Results were amazingly similar for both towns and tend to confirm those obtained for other areas both in Spain and America, basically regarding the fundamental weight of internal factors on $/ \mathrm{d} /$ variation (i.e. consonant maintenance in word-roots vs. deletion in morphemes). However, they contradict most of previous results with regard to the effect of the speaker variables (i.e. younger speakers' leadership on /d/ deletion). Finally, conflict sites reveal sharper social stratification and age differences in Malaga than in Granada.
\end{abstract}

Keywords: sociolinguistics, linguistic variation, quantitative analysis. Andalusian Spanish varieties.

Recibido: 03/06/2016

Aceptado: 01/07/2016 


\section{INTRODUCCIÓN}

En este trabajo se presentan conjuntamente los resultados del estudio de la variación de /d/ intervocálica en la variedad andaluza oriental del español (Málaga y Granada) ${ }^{1}$. Las ventajas de esta investigación consisten en la unificación metodológica en el diseño de las muestras, en el trabajo de campo y en la selección de variables y criterios de organización del estadio analítico (Proyecto PRESEEA). La mayor parte de las hipótesis se avanzaron en los estudios previos de Villena et al. (2011) y Moya Corral y García Wiedemann (2009) sobre el sociolecto de los hablantes universitarios. Lo más interesante es que se confirma la hipótesis central de la investigación comparativa; a saber, las dos comunidades de habla urbanas responden de la misma manera ante la misma variable lingüística; los condicionamientos variables (grupos de factores) sobre la elisión de /d/ son los mismos, están ordenados de la misma forma (misma jerarquía), al igual que las variantes (factores) dentro de cada grupo (Meyerhoff 2009; Tagliamonte 2012). Los elementos incipientes de conflicto (conflict site) suponen aspectos de interpretación de notable interés.

Como ocurre con respecto a otras variables fonológicas, por ejemplo, la /s/ distensiva (Vida Castro 2004; Tejada 2015), las variedades andaluzas se comportan con respecto a la $[ð]$ intervocálica como fuertemente innovadoras. La elisión es un fenómeno no marcado de la fonología del español en todas sus variedades, pero en el español andaluz es mucho más frecuente, si bien parece estar condicionada por los mismos grupos de factores lingüísticos tanto en España (Blas Arroyo 2007; Almeida 2011; Samper 2011; Moya Corral y García Wiedemann 2009, Villena et al. 2011; Villena 2012; Moya et al. 2012; Gómez Molina 2013; Molina y Paredes 2015) como en América (Cedergren 1972; López Morales 1983; D’Introno y Sosa 1986; Alba 1999). El presente estudio aclara en gran medida varios de los puntos más oscuros de investigaciones previas y señala asimismo discrepancias notables entre el área andaluza y el resto de las áreas de España (España centroseptentrional y Canarias).

Del análisis se obtienen algunas conclusiones de interés:

\footnotetext{
1 Los datos y resultados en los que se basa este trabajo se han obtenido en el marco del Proyecto PASOS, FFI2011-29189-C05-01 (Málaga) y 05 (Granada). Se ha beneficiado asimismo de la financiación del Proyecto ECOPASOS, FFI2015-68171-C5-1-P (Málaga) y 2-P (Granada) y fondos FEDER.
} 
1. La elisión es un proceso de cambio lingüístico léxico de sustitución (Moya Corral y García Wiedemann 2009; Villena et al., 2011; Almeida 2011). La difusión del cambio (o su retracción) se produce como condicionada fuertemente por la frecuencia tipo o de inventario (type frequency): morfemas gramaticales en /-ðo/, /-ða/ de los participios verbales y algunas series gramaticales derivadas o analógicamente asimiladas: todo/a, nada (da\#do, da\#da, to( $\varnothing)$ do, to( $\varnothing)$ da). En estos últimos casos, podría pensarse en una extensión del contexto flexional ejemplar (participio) a otros no flexionales pero que comparten rasgos gramaticales y semánticos con él. El efecto de la frecuencia de uso (token frequency) es considerablemente menor, aunque también existe. Aceptaremos aquí la interpretación de la distinción entre frecuencia de inventario (un esquema o patrón representado por muy diversos elementos léxicos) y frecuencia de uso (número de veces que aparece una unidad en un determinado texto), tal y como la propuso y aplicó Bybee (2002) para el mismo fenómeno de elisión de /d/ en español, si bien existen diferentes opciones (Bybee 2007).

2. La mayor parte de las variables internas consideradas como influyentes en los estudios previos (variables del entorno fónico: posición del acento; número de sílabas de la palabra; cualidad de la vocal previa y posterior, etc.) son variables intervinientes cuyo efecto sobre la elisión es solo aparente puesto que está, a su vez, condicionado por la frecuencia de aparición en el corpus de los afijos gramaticales verbales de participio (-do) y los adjetivos y nombres derivados de estos. Al considerar esta circunstancia en el análisis, los modelos probabilísticos de predicción de la elisión de [ð] son más simples y ajustados.

3. Como sucede con bastantes fenómenos fonológicos de no marcación, en Andalucía el cambio está muy generalizado entre los hablantes del nivel educacional más bajo, con un patrón educacional jerárquico y diferencias significativas entre los grupos de edad. Esta circunstancia y el hecho de que los hablantes de edad intermedia y mayores ( $>$ 35 años) de niveles educacionales más altos reaccionen contra el cambio y retengan la $[ð]$ en proporción significativa frente a los más jóvenes (nacidos en los últimos años sesenta), sugiere que en esta área el fenómeno estaba estratificado pero muy extendido y que hoy se desarrolla bien una tendencia conservadora (coincidente con el resto de España) entre los grupos de estatus medioalto, bien una reactivación del cambio entre los más jóvenes y menos instruidos. 
El comportamiento femenino, incluso más contrario al cambio entre los hablantes de más edad, reafirma esta idea.

\section{1. НIPÓTESIS}

La elisión de /d/ es un caso de cambio fónico tendente a facilitar y simplificar la pronunciación natural y no marcada de la aproximante [ð] entre vocales. Esta tendencia se ve favorecida por factores internos gramaticales y del entorno fónico que interaccionan con factores externos que definen los antecedentes y la orientación del hablante hacia el prestigio manifiesto en la comunidad de habla. Ambos conjuntos de factores condicionan (favorecen o restringen) la tendencia a conservar la información completa sobre la forma subyacente de la palabra, lo que permite constatar el efecto decisivo de la frontera morfémica.

Las hipótesis fundamentales son las siguientes:

- La resistencia a la erosión de la estructura lexemática hace que el cambio sea movido o empujado hacia el límite derecho de la palabra, donde se encuentra la información gramatical (morfema: habla\#Do) que es altamente predecible por regla, preservando íntegra la información lexemática (lexema: eDad, humeDad, iDus). La probabilidad de elisión es considerablemente mayor en las palabras bimorfémicas que en las monomorfémicas. La elisión, en consecuencia, viaja a diferente velocidad en el léxico (difusión léxica): se ha generalizado en los sufijos gramaticales pero está relativamente poco avanzada en el cuerpo de las palabras. Esto tiene, evidentemente, que ver con la denominada hipótesis funcionalista (Kiparski 1982; Labov 1994) y con su frecuente refutación en la variación de otros segmentos en español y en otras lenguas (Poplack 1979; Naro 1981; Scherre y Naro 1991, 1997; Vida Castro 2004).

- La elisión en el entorno fónico y gramatical de los participios en \#do (ama\#do, sabi\#do) está muy extendida y tiende a expandirse a las formas derivadas: (adjetivos, nombres) o, incluso, a invadir analógicamente el terreno de los pronombres indefinidos (nada, toda, todo). De este modo, podemos hablar de tres estadios o escalones en la expansión del cambio que determinan tres entornos: participios, derivados morfológicos, lexemas. El efecto de esta distinción se ha comprobado ya en trabajos previos (Villena et al. 2011; Gómez Molina; 2013; Samper 2011; Moya Corral y García Wiedemann 2009, Molina y Paredes 2015). 
- La elisión se manifiesta con mayor frecuencia en el esquema acentual postónico ['daðo], que contrasta con el tónico y el protónico [pe'ðal, aðe'mas]. Los posibles condicionantes fonéticos (intensidad, duración, etc.), sin embargo, interaccionan con la frecuencia de aparición del esquema postónico, que es el propio de los morfemas gramaticales de participio y del de todos sus derivados, que constituyen la combinación de inventario más frecuente en los corpus estudiados.

- El efecto de la estructura de estratificación social de la comunidad de habla interacciona de modo complejo con la jerarquía de difusión del cambio lingüístico, de modo que no todos los hablantes reaccionan igual ante las restricciones sobre este. El ritmo de la restricción del cambio al límite de la palabra (y al entorno fónico -ado) se acelera o se frena según la posición social y las características (edad, sexo) de los hablantes. La hipótesis fundamental es que la elisión de /d/ es un cambio natural y no marcado en su origen que ha afectado a toda /d/ intervocálica desde etapas antiguas en la evolución de la lengua. La necesidad de preservación de las unidades subyacentes (fidelidad) ha interaccionado con la estratificación social de los hablantes propiciando que, con mayor frecuencia, entre los grupos de posición social más alta (élites) se frene la elisión y se restrinja a entornos fonéticos (-ado, -ada) y gramaticales (participios verbales y derivados) predecibles por regla. La elisión se especializa pronto en los sufijos puesto que, al suponer la erosión de material fonético, resulta condicionada por factores como la frecuencia léxica o la redundancia sintagmática (así como por restricciones de fidelidad/ no marcación). Dicho proceso se ordena: (i) en cada variedad (clase, generación, sexo) en función de la frecuencia de inventario (type frequency) y la redundancia (morfemas redundantes (ado) $>>$ morfemas no redundantes $(a d o, a d a) \gg>$ morfemas analógicos (todo, toda, nada); (ii) entre variedades en consonancia con el diferente ritmo del cambio: en los dialectos meridionales el cambio se ha difundido elidiendo casi todas las /d/ en todos los entornos y con frecuencia casi categórica en los más propicios, en tanto que en los dialectos centroseptentrionales está más restringido y es menos frecuente aun en los entornos más propicios. Lo mismo cabe decir de las variedades estilísticas y sociales. Así pues, buena parte de las realizaciones tensas o debilitadas en los dialectos meridionales podrían explicarse como relexificaciones. 
En resumen, la elisión de /d/ combina el efecto de dos factores esencialmente: en primer lugar, la frecuencia de inventario de los elementos léxicos en los que aparece la variable fonológica, que determina mayores probabilidades de elisión en las formas más sometidas al desgaste por ser más usadas. En segundo lugar, la tendencia a la preservación de la forma subyacente del morfema léxico, menos predecible por regla que los morfemas gramaticales. Por un lado, los morfemas en \#ðo, de la primera (a\#) y de la segunda y tercera conjugación (i\#) tienen un uso muy frecuente y un alto grado de predictibilidad (he dao, he cantao); dicha frecuencia aumenta por el amplio número de derivados (adjetivos, nombres): el soldao, la salía, el campo pelao, etc. Por otro lado, se reconoce una resistencia a alterar la raíz de la palabra (constricción de fidelidad).

\section{METODOLOGÍA}

Los corpus respectivos de los que se extrajeron los datos para este trabajo se recogieron, anotaron y editaron de acuerdo con los requerimientos del Proyecto PRESEEA (http://preseea.linguas.net). Las muestras de hablantes estudiadas (Granada, $\mathrm{n}=54$; Málaga, $\mathrm{n}=72$ ) se ajustaron al umbral de la representatividad aconsejada en el mencionado proyecto y se diseñaron a partir de la estratificación por cuotas fijas basada en sexo, edad y nivel educacional, de modo que partimos, respectivamente, de 3 (Granada) y 4 (Málaga) informantes por casilla².

\section{RESULTADOS}

\subsection{FACTORES INTERNOS}

La elisión (36.5 en Granada y 33.6 en Málaga) ocurre mayoritariamente en las formas inflexionadas (respectivamente, 59.2 y 64.7). En las raíces el

\footnotetext{
2 Pueden consultarse los detalles en http://www.vum.uma.es (Málaga) y http:// estudiosdeespanolactual.ugr.es (Granada).
} 
porcentaje baja a 24.3 en Granada y 22.1 en Málaga. Esta elisión lexemática incluye los pronombres y determinantes todo, toda, nada que se comportan como analógicos de las formas dominantes en -ado, -ada. El porcentaje de elisión en Málaga en los lexemas, sin tener en cuenta los mencionados determinantes y pronombres, es solo de 9.1.

Gráfico 1. Elisión de /d/ en Granada y Málaga

Elisión de /d/

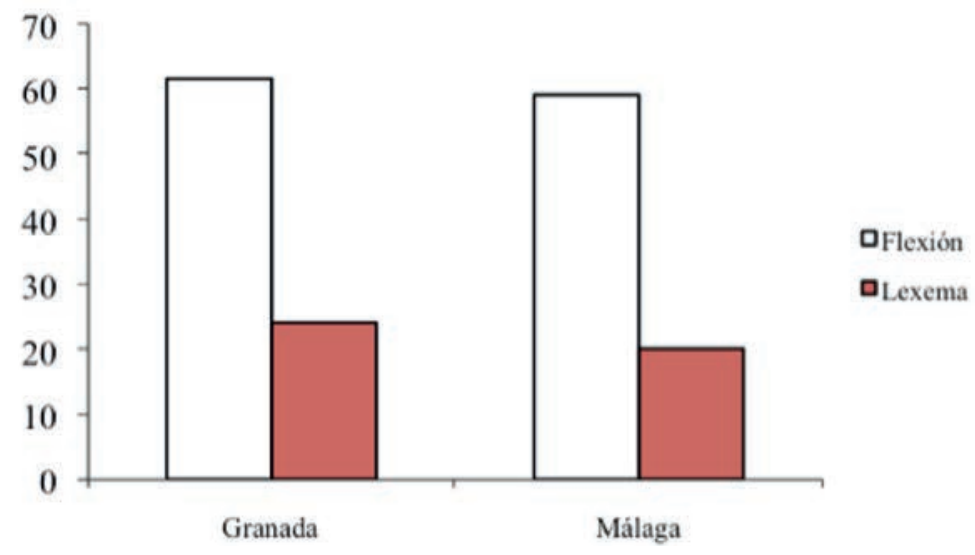

\subsubsection{Entornos vocálicos}

En la Tabla 1 se incluyen los resultados de la elisión de /d/ en los entornos vocálicos en los que su frecuencia es digna de mención. Como se observa son todos entornos de v (tónica)_v, reconocibles como morfemas verbales o adjetivales (ado, ido, ada, ida) o como propios del lexema de los determinantes/pronombres (odo, oda) y de otras categorías (edo, eda). Las combinaciones que no aparecen en la tabla tienen una frecuencia muy baja $(<5.0)$ o corresponden a lexicalizaciones tipo joder, además, heladito (véase 1.2).

Tabla 1. Efecto del entorno vocálico en la elisión de /d/

\begin{tabular}{ccccccc} 
& \multicolumn{3}{c}{ Granada } & \multicolumn{3}{c}{ Málaga } \\
& $\mathrm{n}$ & $\%$ & Total & $\mathrm{n}$ & $\%$ & Total \\
\hline ado & 862 & 86.3 & 999 & 1361 & 85.7 & 1589 \\
oda & 108 & 55.1 & 196 & 104 & 34.9 & 298
\end{tabular}




\begin{tabular}{lcccccc} 
ido & 237 & 48.9 & 485 & 294 & 33.8 & 870 \\
odo & 350 & 46.3 & 756 & 456 & 43.8 & 1040 \\
ada & 284 & 35.8 & 794 & 421 & 34.1 & 1235 \\
ida & 35 & 11,3 & 311 & 28 & 8.9 & 313 \\
eda & 6 & 7.2 & 83 & 17 & 12.4 & 137 \\
edo & 3 & 3.6 & 84 & 6 & 6.7 & 87 \\
\hline
\end{tabular}

Los resultados son muy similares en las dos ciudades. La diferencia está en el avance de la elisión en las terminaciones -ido y -oda en Granada (Gráfico 2).

Gráfico 2. Comparación del efecto del entorno fónico en la elisión de /d/

Elisión de /d/

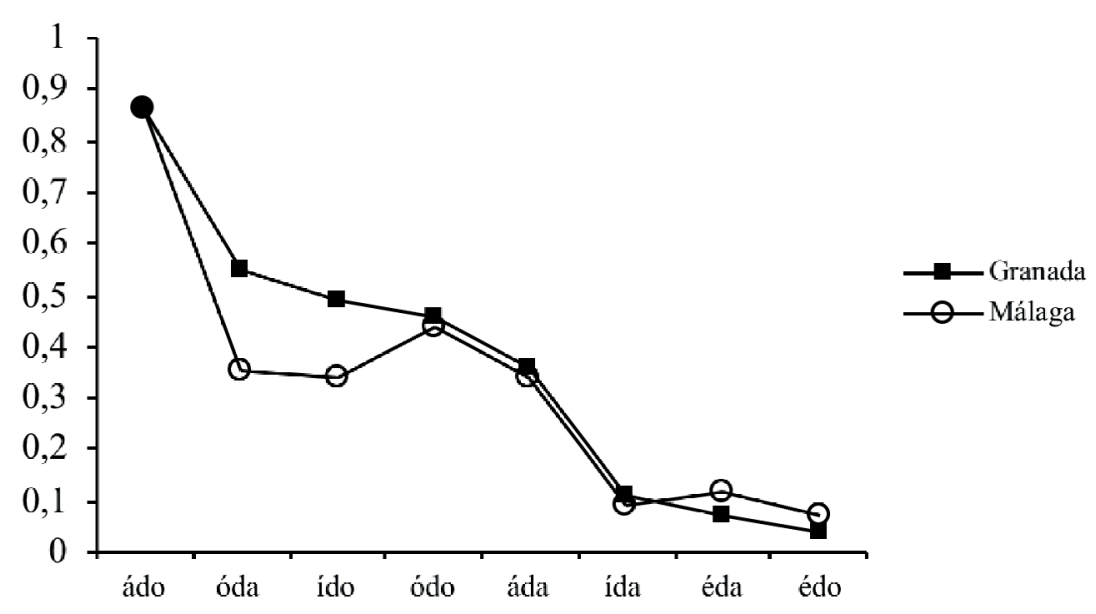

Como se verá después (Sección 3), el avance algo mayor de la elisión en Granada tiene que ver con la incipiente extensión (o resistencia) del cambio más allá del grupo de los hablantes masculinos más jóvenes de estatus bajo (Moya 2012). Que ello se deba a una dilatación de las fronteras de los estilos comunicativos informales entre los hablantes maduros y de estatus intermedio (o jóvenes de estatus bajo), lo que favorecería la elisión en algunos entornos (como -ido, -odo, -oda), es algo que no podemos confirmar de momento. Lo que sí es perceptible es la actual ausencia de tal movimiento en la ciudad de Málaga, donde las fronteras estilísticas y entre grupos etarios y educacionales parecen más firmes. 


\subsubsection{Sílaba}

La hipótesis es que en la elisión de /d/ se da una interacción de tres factores: la categoría morfológica del segmento /d/ en la estructura de la palabra (y, subsecuentemente, el entorno fonológico vocálico), la situación de la sílaba acentuada con respecto a la variable fonológica estudiada y el grado de redundancia. Esto parece estar claro en todos los trabajos en marcha o ya concluidos. Lo que probablemente debería tenerse en cuenta es por qué. La frecuencia de inventario (type frequency) puede ser la clave: las terminaciones morfemáticas (cuya estructura supone, en su gran mayoría, que la sílaba con /d/ es postónica) son con mucho las más frecuentes en los corpus estudiados. De hecho, el 73.0 (6044) de las palabras con /d/ en el corpus de Málaga (8234) tienen la sílaba con /d/ en posición postónica, frente al 24.5 en sílaba tónica o protónica. En Granada, los datos son muy parecidos: 73.8 (5454). Vid. Tabla 2.

Tabla 2. Efecto de la posición del acento y de la frecuencia

(F) del esquema acentual en la elisión de /d/

Granada

\begin{tabular}{cccccc}
\multicolumn{7}{c}{$\begin{array}{c}\text { Granada } \\
\text { No contigua } \\
\text { rápiDo } \\
\text { reDimiré }\end{array}$} & $\begin{array}{c}\text { Postónica } \\
\text { toDo } \\
\text { iDo }\end{array}$ & $\begin{array}{c}\text { Protónica } \\
\text { aDoramos } \\
\text { aDemás }\end{array}$ & $\begin{array}{c}\text { Tónica } \\
\text { peDimos } \\
\text { peDazo }\end{array}$ & Total \\
\cline { 2 - 6 } F & 3.6 & 73.8 & 4.7 & 17.9 & \\
Elisión & 13 & 1892 & 31 & 53 & 2042 \\
$\%$ & 6.6 & 47.0 & 12.2 & 5.4 & 37.4 \\
$\mathrm{n}$ & 197 & 4025 & 254 & 978 & 5454 \\
\hline
\end{tabular}

$\mathrm{Chi}^{2}=740.285(4)$, sig.: $0.000 . \mathrm{V}=0.368$, Sig. 0.000

Málaga

\begin{tabular}{cccccc} 
& $\begin{array}{c}\text { No contiguo } \\
\text { rápiDo } \\
\text { reDimiré }\end{array}$ & $\begin{array}{c}\text { Postónica } \\
\text { toDo } \\
\text { iDo }\end{array}$ & $\begin{array}{c}\text { Protónica } \\
\text { aDoramos } \\
\text { aDemás }\end{array}$ & $\begin{array}{c}\text { Tónica } \\
\text { peDimos } \\
\text { peDazo }\end{array}$ & Total \\
\cline { 2 - 6 } F & 2.0 & 73.4 & 5.9 & 18.6 & \\
Elisión & 2 & 2691 & 25 & 51 & 2769 \\
$\%$ & 1.1 & 44.5 & 5.2 & 3.3 & 33.6 \\
$\mathrm{n}$ & 180 & 6044 & 482 & 1582 & 8234 \\
\cline { 2 - 6 }
\end{tabular}

$\mathrm{Chi}^{2}=1209.627(4)$ sig. $0.000 ; \mathrm{V}=0.383$. Sig. 0.000 
Si bien hay una clara relación entre la elisión de la /d/ postónica y la frecuencia del esquema acentual, se producen dos desajustes, por exceso y por defecto: por un lado, el hasta cierto punto notable número de elisiones (12.2) de la /d/ protónica en Granada, con relación a la escasa frecuencia (4.7) del esquema acentual y, por otro, el exiguo número de elisiones (5.4 en Granada y 3.3 en Málaga) de la /d/ tónica (con respecto a la frecuencia respectiva de dicho esquema acentual: 17.9 y 18.6). El primer caso se explica como consecuencia de las lexicalizaciones de formas elididas de uso frecuente: ADelante, ADemás (16/196, 8.2), ToDavía (11/102, 1.2); el segundo está formado, sobre todo, por derivados diminutivos (afectivos, atenuadores) de participios, adjetivos o nombres en /ado/: diminutivos aDito, aDillo: pescaDito, mercaDillo. Así pues, puede decirse, que, en realidad, la elisión se difunde léxicamente: por un lado, en los morfemas y terminaciones análogas o derivadas; por otro, en ciertas palabras que han fijado la forma elidida. Como se observa en la Tabla 3, el comportamiento de las palabras con /d/ en sílaba tónica (poDer, queDar, alredeDor) es bastante variable, como ocurre asimismo con las sílabas postónicas (viDa, boDa) y protónicas (toDavía, aDemás, aDelante).

Tabla 3. Difusión léxica de la elisión de /d/

\begin{tabular}{|c|c|c|c|c|c|c|c|c|c|}
\hline & & ToDaví & Demá & Delante & $\mathrm{ViDa}$ & BoDa & PoDer & QueDar & AlredeDor \\
\hline \multirow[t]{3}{*}{ Málaga } & $\mathrm{n}$ & 102 & 196 & 14 & 134 & 28 & 437 & 272 & 17 \\
\hline & Elisión & 11 & 16 & 10 & 1 & 0 & 16 & 46 & 0 \\
\hline & $\%$ & 1.2 & 8.2 & 71.4 & $\begin{array}{l}0.0 \\
0.0\end{array}$ & 0.0 & 3.7 & 16.9 & 0.0 \\
\hline \multirow[t]{3}{*}{ Granada } & $\mathrm{n}$ & 48 & 110 & 7 & 127 & 8 & 353 & 185 & 26 \\
\hline & Elisión & 11 & 37 & 1 & 1 & 0 & 15 & 36 & 1 \\
\hline & $\%$ & 22.9 & 33.6 & 14.3 & 0.8 & 0.0 & 4.2 & 19.5 & 5.0 \\
\hline
\end{tabular}

En consecuencia, la posición del acento no explica toda la variación. Sería necesario entonces considerar factores al margen de este; esto es, la interacción de la terminación y la categoría morfológica. En todo caso, es probable que sea la propia naturaleza de la sílaba -su carácter tónico- lo que frene la elisión esperable. Los datos se pueden interpretar de dos formas diferentes:

1. Oposición entre/d/tónica (helaDito, poDer) y /d/átona (toDo, rápiDo, reDimiré, aDoramos) sin tener en cuenta la posición antepuesta o pospuesta del acento (Almeida 2011). Esta opción mezcla el entorno 
de /d/ postónica muy frecuente y propicio a la elisión (cantaDo, $n a D a, t o D o$ ) con uno (el de /d/ protónica y el de /d/ no contigua) poco frecuente y poco propicio a la elisión (rápiDo, reDimiré, aDoramos). Tiene, sin embargo, la ventaja de que puede explicarse por razones fonéticas ligadas a la tensión, duración, tono alto, etc. de los sonidos acentuados frente a los átonos (Tabla 4).

2. Oposición entre la/d/ precedida por el acento (cantaDo) o seguida por el acento en la misma (poDer) o en la sílaba siguiente (aDoramos). La ventaja es que la posición posterior del acento retiene el segmento frente a la posición anterior, que favorece su elisión. La desventaja es que se pierde la base de la explicación fonética.

Tabla 4. Efecto del carácter tónico/átono de la /d/ en Málaga y Granada

\begin{tabular}{ccccccc}
\multicolumn{3}{c}{ Málaga } & \multicolumn{3}{c}{ Granada } \\
\hline & Átona & Tónica & Total & Átona & Tónica & Total \\
$\mathrm{d}$ & 3810 & 1477 & 5287 & 2541 & 925 & 3466 \\
$\%$ & 58.4 & 96.7 & 65.6 & 56.8 & 94.6 & 63.5 \\
Elisión & 2716 & 51 & 2767 & 1936 & 53 & 1989 \\
$\%$ & 41.6 & 3.3 & 34.4 & 43.2 & 5.4 & 36.5 \\
Total & 6526 & 1528 & 8054 & 4477 & 978 & 5455 \\
\hline
\end{tabular}

Málaga, $\mathrm{Chi}^{2}=804.484$, sig. 0.000; Phi $=-0.316$, sig. $0.000 / \mathrm{Granada}_{\mathrm{Chi}}{ }^{2}=530.012$, sig. 0.000; Phi $=-0.318$, sig. 0.000

Átona: daDo, aDelante (se eliminaron las sílabas átonas con acento mediato)

Tónica: peDimos

Como se observa, la sílaba tónica retiene el segmento (96.7 y 94.6 en Málaga y Granada) a pesar de su frecuencia respectiva relativamente alta en los corpus estudiados (18.6 y 17.9).

\subsubsection{Frecuencia y elisión}

La sílaba postónica favorece la elisión. Sin embargo, no en todos los entornos por igual, como se observó arriba (1.1). Ahora bien, como demuestran los datos, se da una relación positiva entre, por un lado, la frecuencia de aparición de los esquemas acentuales y de entorno vocálico (frecuencia de inventario o type frequency) y, por otro, la frecuencia de elisión, independientemente de qué forma léxica los represente en el corpus. En la Tabla 5 se muestra el porcentaje de elisión (e-0) en los diferentes entornos vocálicos/acentuales, junto con la frecuencia de aparición (F) de cada uno de ellos en el corpus estudiado. Se añade asimismo el peso que la elisión tiene en cada entorno 
con relación al número total de elisiones en el corpus de datos (e-0/corpus) y el peso acumulado (e-0/acum./corpus).

Lo más interesante es que el porcentaje acumulado en el corpus de la elisión de /d/ postónica (1-6) está por encima del 94\% (96.3 en Málaga y 94.3 en Granada), lo que significa que solo el 3.7 y $5.7 \%$, respectivamente, de la elisión se produce en sílaba tónica o protónica. Como se observó en el párrafo anterior, las circunstancias en las que las sílabas cuyo ataque es /d/ están más frecuentemente expuestas a la erosión (y, por lo tanto, esta tiene una elevada probabilidad de producirse), son las siguientes: 1) factor morfológico: terminaciones morfemáticas (vs. lexema);2) posición postónica (vs.) protónica o tónica. Este hecho: que las sílabas postónicas con /d/ (que coinciden con que son morfemas de los participios o terminaciones asimiladas a estos) aparecen más en el corpus que ningún otro morfema léxico o gramatical con /d/ está en la base de la explicación de por qué se produce tan elevada frecuencia de elisión. La frecuencia acumulada en el corpus de estas formas con/d/ en sílaba postónica es casi exacta en Granada (65.0) y Málaga (64.9). Esto quiere decir que el 65\% de las palabras en las que la /d/ se expone al desgaste fonético tienen la estructura: v[acento]_v. El resto corresponde a entornos con /d/ en sílaba tónica, /d/ protónica o a combinaciones con frecuencia muy baja $(<0.9 \%)$. Como se verá enseguida (1.4), la estructura fónica viene, en realidad, impuesta por la categoría morfológica.

Tabla 5. Frecuencia de inventario y de elisión de /d/ en Málaga y Granada

\begin{tabular}{|c|c|c|c|c|c|c|c|c|}
\hline & & & & Mál & & & & \\
\hline & Entornos & Ejemplo & $\mathrm{F}$ & $\mathrm{n}$ & $\mathrm{e}-0$ & e- $0 /$ corpus & $\begin{array}{l}\text { e- } 0 \text { acum./ } \\
\text { corpus }\end{array}$ & $\mathrm{n}$ \\
\hline 1 & ádo & hablado & 19.3 & 1361 & 85.7 & 49.2 & 49.2 & 1589 \\
\hline 2 & áda & helada, nada & 15.0 & 421 & 34.1 & 15.2 & 64.4 & 1235 \\
\hline 3 & ódo & todo & 12.6 & 456 & 43.8 & 16.5 & 80.9 & 1040 \\
\hline 4 & ído & comido & 10.6 & 294 & 33.8 & 10.6 & 91.5 & 870 \\
\hline 5 & ída & salida & 3.8 & 28 & 8.9 & 1.0 & 92.5 & 313 \\
\hline 6 & óda & toda & 3.6 & 104 & 34.9 & 3.8 & 96.3 & 298 \\
\hline 7 & ade & adelante & 2.4 & 16 & 8.2 & 0.6 & 96.9 & 196 \\
\hline 8 & oda & todavía & 1.3 & 12 & 11.4 & 0.4 & 97.3 & 105 \\
\hline 9 & adí & heladito & 1.1 & 27 & 30.7 & 1.0 & 98.3 & 88 \\
\hline 10 & odé & joder & 0.9 & 9 & 11.8 & 0.3 & 98.6 & 76 \\
\hline 11 & resto & & 29.4 & 41 & 1.7 & 1.4 & 100.0 & 2424 \\
\hline & Total & & 100.0 & 2769 & 100.0 & & & 8234 \\
\hline
\end{tabular}




\begin{tabular}{|c|c|c|c|c|c|c|c|c|}
\hline & & & & Grat & & & & \\
\hline & Entornos & Ejemplo & $\mathrm{F}$ & $\mathrm{n}$ & $e-0$ & e- $0 /$ corpus & $\begin{array}{c}0 \text {-e acum./ } \\
\text { corpus }\end{array}$ & $\mathrm{n}$ \\
\hline 1 & ádo & hablado & 18.3 & 862 & 86.3 & 43.3 & 43.3 & 999 \\
\hline 2 & áda & helada, nada & 14.6 & 284 & 35.8 & 14.3 & 57.6 & 794 \\
\hline 3 & ódo & todo & 13.9 & 350 & 46.3 & 17.6 & 75.2 & 756 \\
\hline 4 & ído & comido & 8.9 & 237 & 48.9 & 11.9 & 87.1 & 485 \\
\hline 5 & ída & salida & 5.7 & 35 & 11.3 & 1.8 & 88.9 & 311 \\
\hline 6 & óda & toda & 3.6 & 108 & 55.1 & 5.4 & 94.3 & 196 \\
\hline 7 & ade & adelante & 2,3 & 21 & $16.7^{-}$ & 1.1 & 95.4 & $126^{-}$ \\
\hline 8 & oda & todavía & ,9 & 8 & 15.7 & 0.4 & 95.8 & 51 \\
\hline 9 & adí & heladito & 1,3 & 34 & 47.2 & 1.7 & 97.2 & 72 \\
\hline 10 & odé & joder & 1,2 & 2 & 2.9 & 0.1 & 97.3 & 68 \\
\hline 11 & resto & & 29.3 & 48 & 3.0 & 2.4 & 100.0 & 1597 \\
\hline & Total & & 100.0 & 1989 & & 100.0 & & \\
\hline
\end{tabular}

\subsubsection{Interacción morfofonológica}

Como es bien sabido, existe una clara jerarquía morfológica en la frecuencia de elisión que indica que la mayor parte de la elisión de /d/ se produce en los participios, en los adjetivos y en los pronombres y determinantes (frecuencia acumulada en el corpus: 2379/2769, 86.0 en Málaga y 1642/1989, 82.6 en Granada). En la Tabla 6 se muestran los datos. La única alteración del orden está en el porcentaje relativamente elevado de elisión en los adverbios en Granada (donde se incluyeron como adverbios casos de todo).

Tabla 6. Efecto de la categoría morfológica en la elisión de /d/

Granada Málaga

Categ. Frec. $\% \quad \% \quad \% \quad$ Total Categ. Frec. $\% \quad \% \quad \%$ Total corpus acum. corpus acum.

\begin{tabular}{lc|cccccccccc}
\hline Part. & 762 & 75.1 & 38.3 & 38.3 & 1015 & Part. & 1062 & 70.1 & 38.4 & 38.4 & 1514 \\
Adj. & 302 & 49.3 & 15.2 & 53.5 & 612 & Adj & 473 & 50.4 & 17.1 & 55.5 & 939 \\
Pro & 578 & 46.8 & 29.1 & 82.6 & 1236 & Pro & 844 & 41.5 & 30.5 & 86.0 & 2035 \\
\hline Adv & 68 & 22.1 & 12.2 & 94.8 & 308 & N & 322 & 13.7 & 11.6 & 97.6 & 2348 \\
N & 243 & 15.5 & 3.5 & 98.2 & 1563 & Adv & 30 & 2.8 & 1.3 & 98.9 & 1057 \\
V & 31 & 4.5 & 1.7 & 100.0 & 696 & V & 29 & 8.9 & 1.1 & 100.0 & 326 \\
Total & 1989 & 36.5 & & & 5455 & Total & 2769 & 33.6 & & & 8234 \\
\hline
\end{tabular}

Chi $^{2}$ Granada: 1388.320 , sig. $0.000 ; \mathrm{V}=0.505$, sig. 0.000

$\mathrm{Chi}^{2}$ Málaga: 2038.791, sig. 0.000; $\mathrm{V}=0.498$, sig. 0.000 
Hasta aquí se ha observado, en primer lugar, que la posición fónica de la sílaba en la que la elisión de /d/ es más frecuente es la postónica y se ha puesto de manifiesto que es esta posición en la que aparece con mayor frecuencia dicha obstruyente (Tabla 2). En segundo lugar, se ha constatado que la elisión de /d/ está relacionada con dicha frecuencia y que en la sílaba postónica aparecen las terminaciones propias de morfemas gramaticales de participio $\mathrm{y}$ de ciertos determinantes y pronombres de uso frecuente. En tercer lugar, se ha comprobado la baja frecuencia de la elisión en la sílaba tónica a pesar de que su frecuencia de uso es notable (18.6 y 17.9), lo que puede ser explicado por la mayor tensión, duración e intensidad de la sílaba tónica frente a la átona. En cuarto lugar, se ha observado que la frecuencia de elisión está relacionada con la frecuencia de aparición de los entornos vocálicos (Tabla 5) y, dado que no existe hasta ahora un fundamento fonético que explique la diferente tasa de elisión en los diferentes entornos vocálicos (ado >> ido, etc.), la hipótesis, en la línea mencionada arriba de los estudios de Bybee (2002, 2007), Pierrehumbert (2001) y otros, es que dicha tasa depende de la frecuencia de aparición de las terminaciones (Tabla 5).

Si se considera ahora la distribución de las terminaciones y la categoría gramatical en un grupo interactivo (Tabla 7), se observa una jerarquía interna dentro de cada entorno vocálico que, a falta de una razón fonética, se explica por la frecuencia de las distintas categorías gramaticales. En la tabla se indica, por un lado, la frecuencia de aparición en el corpus de cada grupo interactivo gramatical/fónico (Fgrupo), junto con la frecuencia del entorno (Fent.); por otro lado, la frecuencia de elisión en el grupo interactivo (\% grupo), en la categoría gramatical (\% categ.) y en el entorno fónico (\% ent.). Los datos que se exponen corresponden solo al corpus de Málaga puesto que los de Granada son muy similares.

La ordenación de la elisión -establecida según el porcentaje de elisión en el entorno- solo se altera por el uso desproporcionadamente alto de -oda (toda), que se explica por razones morfológicas: el 100.0 de los casos de elisión se debe al pronombre/det. toda (los 53 casos restantes son nombres que retienen la /d/); esta última forma elide la /d/ por analogía con el masculino todo, cuyo uso es muy superior (12.6). Con respecto al clasema [humano], la hipótesis es que la elisión es más frecuente en los sufijos que en la raíz de la palabra, pero que puede suponerse una predisposición mayor en los sufijos de los participios en tiempos compuestos, adjetivos y nombres [+ humano] que en los nombres [- humano] y en los nombres y adjetivos con sufijo derivativo. La frecuencia media relativamente alta de elisión en los nombres no humanos incluye -ador, -adora, -idor, etc. y -adillo, -adito (heladillo, puñadillo, ladillo, jodidillas, agobiadillas, escapadilla, sudadico, etc.). 
Tabla 7. Condicionamiento gramatical y fónico de la elisión de /d/ en Málaga

\begin{tabular}{|c|c|c|c|c|c|c|c|c|c|}
\hline Ent. & Categ. & Ejemplo & Fgrupo & Fent. & Elisión & $\%$ ent. & $\begin{array}{c}\% \\
\text { grupo }\end{array}$ & $\begin{array}{c}\% \\
\text { categ. }\end{array}$ & Total \\
\hline ado & Part\# & he hablado & 10.7 & & 812 & & 92.0 & 59.7 & 883 \\
\hline ado & $\mathrm{A}, \mathrm{N}(+\mathrm{H})$ & un soldado & 8.3 & 19.3 & 333 & 85.7 & 78.5 & 24.5 & 424 \\
\hline ado & $\mathrm{N}(-\mathrm{H})$ & un candado & 0.8 & & 45 & & 70.3 & 3.3 & 64 \\
\hline odo & P,Det0 & todo & 12.6 & 12.6 & 451 & 34.8 & 43.7 & 98.9 & 1033 \\
\hline oda & P,Det0 & toda & 2.9 & 3.6 & 104 & 34.9 & 42.4 & 100.0 & 245 \\
\hline ada & $\mathrm{A}, \mathrm{N}(+\mathrm{H})$ & está salada & 6.0 & & 105 & & 41.3 & 29.4 & 254 \\
\hline ada & P,Det0 & nada & 8.7 & 15.0 & 715 & 34.1 & 37.6 & 63.9 & 269 \\
\hline ada & $\mathrm{N}(-\mathrm{H})$ & una panzada & 0.7 & & 8 & & 13.6 & 1.9 & 59 \\
\hline ido & Part\# & he salido & 7.6 & & 243 & & 38.9 & 82.7 & 624 \\
\hline ido & $\mathrm{A}, \mathrm{N}(+\mathrm{H})$ & un salido & 1.7 & 10.6 & 31 & 33.8 & 24.8 & 10.5 & 125 \\
\hline ido & $\mathrm{N}(-\mathrm{H})$ & un partido & 0.5 & & 7 & & 16.3 & 2.4 & 43 \\
\hline ida & $\mathrm{A}, \mathrm{N}(+\mathrm{H})$ & está perdida & 3.3 & & 13 & 89 & 26.0 & 46.4 & 50 \\
\hline ida & $\mathrm{N}(-\mathrm{H})$ & la herida & 1.0 & 5.6 & 13 & 0.9 & 16.5 & 46.4 & 79 \\
\hline
\end{tabular}

Se puede concluir que, aunque se noten diferencias en el porcentaje de elisión entre los diferentes grupos interactivos, es muy probable que los hablantes operen, perciban y se representen la variación basada en los entornos: -ado (85.7), -odo, -ada, -ido $(\approx 34.0)$ y resto. La frecuencia de inventario se postula pues como una variable predictora del máximo peso, tal y como se demuestra más adelante (Sección 3.3).

\subsubsection{Frecuencia de uso y frecuencia de inventario}

Desde los trabajos de Bybee (2002) se sabe que la frecuencia de uso de las palabras en un corpus dado (token frequency) impulsa los cambios fónicos de reducción puesto que expone las variables susceptibles de debilitarse y desaparecer a un mayor riesgo de desgaste, frente a lo que ocurre con los cambios analógicos, que se extienden primero en las palabras de baja frecuencia. Sin embargo, este factor interacciona con otro: el contexto que promueve la reducción, esto es, el entorno en el que el cambio se produce con mayor frecuencia (type frequency). Sería de esperar, entonces, que: 1) las palabras más frecuentes elidiesen con mayor frecuencia la /d/ que las menos frecuentes; 2) el contexto de uso promotor de la reducción (los participios usados como formas verbales o como adjetivos, nombres, etc.) multiplicase la reducción, dada asimismo su alta frecuencia. En nuestro caso, como se vio arriba (Tabla 6), la frecuencia acumulada de elisión en 
las formas flexionadas (Adj, Part) está entre 53.00 y 56.00. Si se añaden los determinantes y pronombres (todo, toda, nada), a los que parece extenderse el cambio por asimilación al núcleo ejemplar, la frecuencia acumulada sube (82.6 en Granada y 86.0 en Málaga).

La primera hipótesis se cumple con cierta claridad, en concurrencia con los resultados de trabajos previos (Bybee 2002: 265-267 et passim; Almeida 2011). En la Tabla 8 se muestra el efecto de la frecuencia de uso en la elisión de /d/. Se han utilizado los datos del léxico de frecuencia del español hablado en la ciudad de Málaga (Ávila Muñoz 1999: 137-340). Dado que la frecuencia de las ocurrencias y lemas en el diccionario se calcula sobre el total de formas en el corpus original, las cifras reales se multiplicaron por un millón, de modo que fuese más inmediata la captación de los valores (ibid., 135, n. 2). Como puede observarse, la token frequency tiene un efecto débil $(\mathrm{V}=0.141)$ pero significativo $\left(\mathrm{Chi}^{2}: 7242,2=143.793\right.$, sig. $\left.=0.000\right)$ sobre la elisión en el sentido esperado: en las palabras más frecuentes (frec. $>$ 1000) es más frecuente la elisión de /d/ (40.2) que en las menos frecuentes: $500-1000(30.4)$ y $<500(26.2)$.

Tabla 8. Efecto de la frecuencia de uso de las formas léxicas en la elisión de /d/ en Málaga

\begin{tabular}{ccccc}
\multicolumn{5}{c}{ Frecuencia } \\
& $0-500$ & $500-1000$ & $>1000$ & Total \\
\hline $\mathrm{d}$ & 2228 & 577 & 2028 & 4833 \\
& 73.8 & 69.6 & 59.8 & 66.7 \\
0 & 793 & 252 & 1364 & 2409 \\
\multirow{2}{*}{ Total } & 26.2 & 30.4 & 40.2 & 33.3 \\
& 3021 & 829 & 3392 & 7242 \\
\hline
\end{tabular}

$\mathrm{Chi}^{2}=143.793$ (2); sig. $=0.000 ; \mathrm{V}=0.141$. sig. 0.000

Casos perdidos $=992(12.0)$.

En cuanto a la segunda hipótesis, los datos de Málaga ponen de manifiesto que la frecuencia de uso de la palabra predice la elisión en el lexema, pero que no influye significativamente en la elisión morfemática (Tabla 9). La elisión en los lexemas sube conforme sube la frecuencia de las palabras, pero no hay relación entre esta y la frecuencia de elisión en el morfema. Esto quiere decir que el hecho de que el morfema \#aDo se combine, por ejemplo, con una palabra muy frecuente, como dar (2697.7) o con una de baja frecuencia, como remunerar (0.0) no influye en la tasa de elisión en el sufijo; pero la frecuencia de la palabra sí que determina la tasa de elisión 
en la raíz: deDo, meDa. Así pues, la elisión es mucho más frecuente en los morfemas (66.7) que en los lexemas (23.7), pero en los morfemas no hay variación significativa como efecto de la frecuencia y sí en los lexemas.

Tabla 9. Interacción de la frecuencia de uso y de la frontera morfémica en la elisión de /d/ en Málaga

\begin{tabular}{lccccc} 
& & \multicolumn{3}{c}{ Frecuecia } & \\
& & $0-500$ & $500-1000$ & $>1000$ & Total \\
\hline \multirow{3}{*}{ Lexema } & $\mathrm{d}$ & 2150 & 562 & 1997 & 4709 \\
& & 89.4 & 77.6 & 66.3 & 76.7 \\
& 0 & 254 & 162 & 1016 & 1432 \\
& & 10.6 & 22.4 & 33.7 & 23.7 \\
& Total & 2404 & 724 & 3013 & 6141 \\
& $\mathrm{~d}$ & 78 & 15 & 31 & 124 \\
Morfema & & 12.6 & 14.3 & 8.2 & 11.3 \\
& 0 & 539 & 90 & 348 & 977 \\
& & 87.4 & 85.7 & 91.8 & 88.7 \\
& Total & 617 & 105 & 379 & 1101 \\
& $\mathrm{~d}$ & 2228 & 577 & 2028 & 4833 \\
& & 73.8 & 69.6 & 59.8 & 66.7 \\
& 0 & 793 & 252 & 1364 & 2409 \\
& & 26.2 & 30.4 & 40.2 & 33.3 \\
& & 3021 & 829 & 3392 & 7242 \\
\hline
\end{tabular}

Lexema, Chi²: 6141, $2=401.334$, sig. $=0.000 ; \mathrm{V}=0.356$, sig. $=0.000$ Morfema, $\mathrm{Chi}^{2}=1101,2=5.739$, sig. $0.057 ; \mathrm{V}=0.072$, sig. $=0.057$

Los resultados en esta primera aproximación aunque corroboran parcialmente resultados previos, tienden a limitarlos considerablemente: basándonos, al menos, en los datos de Málaga, solo se puede afirmar que la elisión aumenta conforme aumenta la frecuencia léxica en las raíces de las palabras pero no en los morfemas. Es muy probable que esto tenga que ver con la generalización del cambio, que se ha extendido en la flexión y se ha detenido en las raíces (Gráfico 3). 
Gráfico 3. Estratificación educacional y límite morfémico en la elisión de /d/

\section{Elisión de/d/}

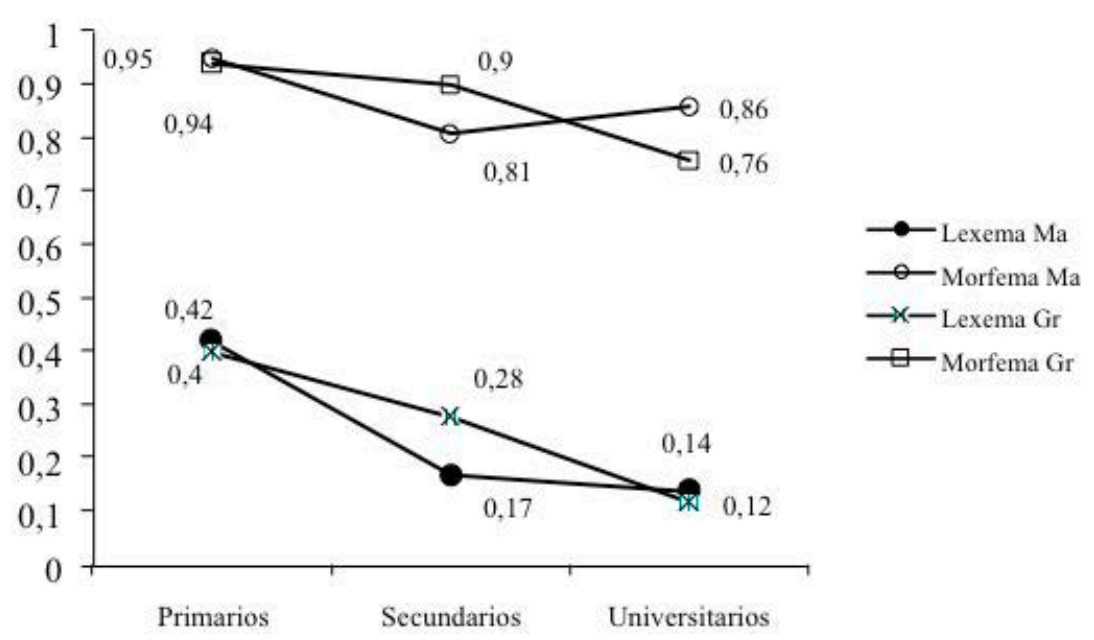

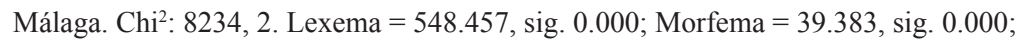
Total $=514.008$, sig. 0.000

Granada. Chi $^{2}: 5455,2$. Lexema $=304.335$, sig. $=0.000 ;$ Morfema $=55.841$, sig. 0.000; Total $=309.280$, sig. 0.000

La frecuencia léxica tiene, pues, efecto sobre la elisión en los lexemas porque el cambio está poco avanzado, pero en los entornos ejemplares del cambio (morfemas) tal efecto no existe. Ambos entornos parecen discurrir separadamente, si bien se distribuyen de manera similar en la estratificación educacional en ambas ciudades.

\subsection{Factores externos}

Las tasas de elisión en las dos ciudades son parecidas (Tabla 10); las diferencias más importantes provienen (véase 3.1.1) del mayor avance en Granada de la elisión en los participios en -ido y en el det/pro toda (sílaba postónica). En la Tabla 10 y en el Gráfico 4 se muestran los resultados separados en cuatro entornos teniendo en cuenta el efecto de la posición del acento y su combinación con la presencia de límite morfémico en la palabra (incluyendo los participios y adjetivos derivados) y con la cualidad de las vocales previa y posterior. El entorno general incluye todas las ocurrencias 
en el corpus; el postónico limita estas a la sílaba postónica inmediata al acento y excluye todas las demás; el bimorfémico elimina las ocurrencias $\mathrm{de} / \mathrm{d} /$ en los lexemas y, por último, Ado computa únicamente los participios en construcciones verbales.

Tabla 10. Frecuencia de elisión de /d/ en los entornos

\begin{tabular}{llclclclc}
\multicolumn{2}{l}{ Todos } & \multicolumn{2}{l}{ Postónica } & \multicolumn{3}{l}{ Bimorfémico } & \multicolumn{2}{l}{ Ado } \\
\hline & Elisión & $\%$ & Elisión & $\%$ & Elisión & $\%$ & Elisión & $\%$ \\
Granada & $1989 / 5455$ & 36.5 & $1889 / 1987$ & 47.4 & $1118 / 1566$ & 59.2 & $546 / 595$ & 91.8 \\
Málaga & $2769 / 8234$ & 33.6 & $2691 / 6044$ & 44.5 & $1888 / 2652$ & 61.6 & $812 / 883$ & 92.0 \\
\hline
\end{tabular}

Gráfico 4. Entornos de elisión de /d/ en Andalucía

Elisión de /d/

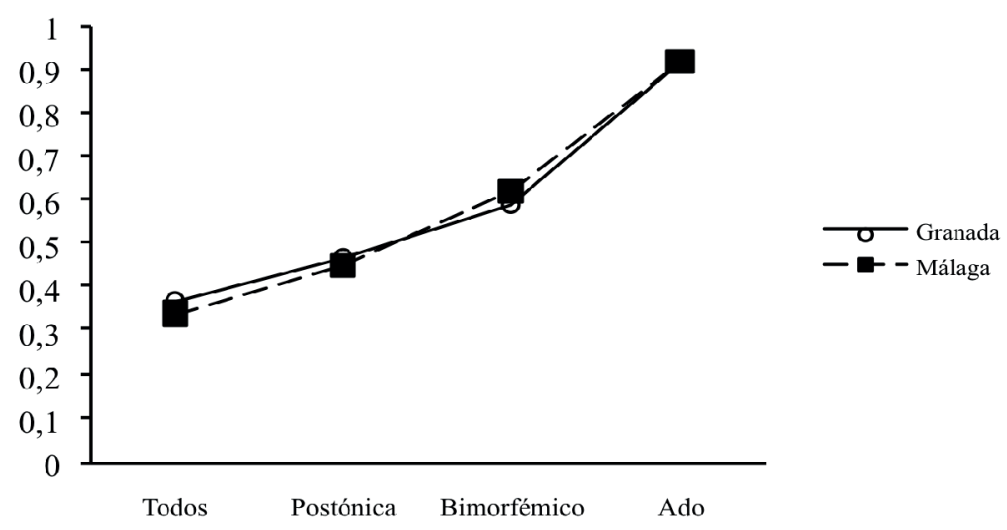

\subsubsection{Estilo y otras dimensiones diafásicas}

La formalidad de la actuación lingüística de los hablantes, al menos tal y como fue medida aquí a través de la modificación del rol del interlocutor en diversas dimensiones (estatus, edad, grado de proximidad), no tiene efecto significativo en la elisión de /d/; sin embargo, el grado de marcación de las formas léxicas empleadas, el género discursivo e, incluso, el priming o efecto mecánico de la ocurrencia de la variable en la misma palabra, en la secuencia o en el turno anterior sí la determinan. El efecto de todos estos factores es significativo, si bien su intensidad no es grande. Estamos aquí, probablemente, ante la consecuencia indeseada de la estructura misma de 
la investigación (entrevista semidirigida con un esquema conversacional definido de antemano) puesto que la dimensión diafásica (especialmente el estilo) debe de tener seguramente mayor influencia que la que se refleja aquí.

\subsubsection{Género discursivo}

La interacción dialogada espontánea al margen de la estructura de pares adyacentes típica de la entrevista semidirigida favorece la elisión en ambas ciudades. Estos intercambios espontáneos surgen irregular pero frecuentemente a lo largo de la entrevista y rompen el esquema regular de pregunta y respuesta. Del mismo modo, cuando el informante lleva a cabo una narración, una descripción o una argumentación, al margen de la estructura de pares adyacentes de la entrevista, retiene asimismo la /d/ en mayor medida que en los coloquios espontáneos (Tabla 11). El efecto de esta variable es -junto con la marcación léxica- el más importante, comparativamente, de los correspondientes a las variables incluidas en este grupo "diafásico", como se verá más adelante (Sección 3.3).

Tabla 11. Efecto del género discursivo en la elisión de /d/

\begin{tabular}{lcccccc}
\multicolumn{5}{c}{ Málaga } & \multicolumn{2}{c}{ Granada } \\
\hline \multirow{2}{*}{$\mathrm{d}$} & Diálogo & Otros & Total & Diálogo & Otros & Total \\
& 1869 & 3596 & 5465 & 1160 & 2306 & 3466 \\
0 & 57.5 & 72.1 & 66.4 & 57.8 & 66.9 & 63.5 \\
0 & 1380 & 1389 & 2769 & 846 & 1143 & 1989 \\
\multirow{2}{*}{ Total } & 42.5 & 27.9 & 33.6 & 42.2 & 33.1 & 36.5 \\
\cline { 2 - 7 } & 3249 & 4985 & 8234 & 2006 & 3449 & 5455 \\
\hline
\end{tabular}

Málaga. Chi²: $^{2} 234,1=188.137$, sig. $0.000, \mathrm{Phi}=-0.151$

Granada. Chi' $: 5455,1=44.075$, sig. $0.000, \mathrm{Phi}=0.090$

\subsubsection{Priming}

La tendencia a la repetición (priming) de estructuras previamente emitidas (por el emisor o por otros participantes en el acontecimiento de habla) o a la anticipación de las previstas constituye un efecto mecánico del procesamiento del lenguaje comprobado en variadas direcciones y en todos los componentes lingüísticos (Almeida 2009: 22-31). Este efecto mecánico parece influir aquí con claridad tanto en el marco de la estructura secuencial próxima a la variable dentro del mismo turno, como en el efecto del turno anterior; en ambos casos, aumenta la frecuencia de retención del segmento si este se retuvo previamente. Sin embargo, existen ciertas restricciones y dudas en lo referente al efecto mecánico en el interior de la palabra. 
En primer lugar, el efecto de priming se manifiesta en el aumento de la frecuencia de retención del segmento cuando en el turno anterior se han producido una o varias ocurrencias de /d/ realizada como alófono tenso [ð]. Las diferencias son significativas si bien el efecto es débil (Tabla 12).

Tabla 12. Efecto de priming del turno anterior en la variación de /d/

\begin{tabular}{lcccccccc} 
& \multicolumn{3}{c}{ Granada } & \multicolumn{5}{c}{ Málaga } \\
$\begin{array}{lcccccccc}\text { Turno } \\
\text { anterior }\end{array}$ & 0 & {$[\mathrm{~d}]$} & No & Total & 0 & {$[\mathrm{~d}]$} & No & Total \\
\hline \multirow{3}{*}{ Elisión } & 63 & 156 & 1770 & 1989 & 314 & 344 & 2019 & 2767 \\
& 13.2 & 21.9 & 38.9 & 36.5 & 50.0 & 27.2 & 33.3 & 33.6 \\
& 190 & 711 & 4553 & 5454 & 628 & 1263 & 6334 & 8225 \\
\hline
\end{tabular}

Granada. $\mathrm{Chi}^{2}=77.050$ (3), sig. $0.000 . \mathrm{V}=0.119$, sig. 0.000

Málaga. $\mathrm{Chi}^{2}=99.368(3)$, sig. $0.000 . \mathrm{V}=0.110$, sig. 0.000

En segundo lugar, el efecto mecánico de la retención de /d/ antes o detrás de la palabra en la que está la variable también parece claro, puesto que, cuando se retiene la/d/ (me[ð]i[ð]as observa/d/as) el porcentaje aumenta en más de diez puntos por encima del que existe cuando no hay otra/d/ en la secuencia $\mathrm{y}$ en casi treinta puntos cuando hay instancias de /d/ en la secuencia (la ha cortao to/d/a) y estas se eliden (Tabla 13).

Tabla 13. Efecto de priming en la secuencia sobre la elisión de /d/

\begin{tabular}{|c|c|c|c|c|c|c|c|c|}
\hline & \multicolumn{5}{|c|}{ Málaga } & \multicolumn{3}{|c|}{ Granada } \\
\hline & No ocurr. & [ð] & 0 & Total & No ocurr. & [ð] & 0 & Total \\
\hline \multirow[t]{2}{*}{ d } & 4031 & 1084 & 317 & 5432 & 2230 & 883 & 345 & 3458 \\
\hline & 65.6 & 78.7 & 49.1 & 66.5 & 63.2 & 75.5 & 46.3 & 63.5 \\
\hline \multirow[t]{2}{*}{0} & 2114 & 294 & 328 & 2736 & 1298 & 287 & 400 & 1985 \\
\hline & 34.4 & 21.3 & 50.9 & 33.5 & 36.8 & 24.5 & 53.7 & 36.5 \\
\hline Total & 6145 & 1378 & 645 & 8168 & 3528 & 1170 & 745 & 5443 \\
\hline $\begin{array}{l}\text { Chi }^{2}: 8 \\
V=0.1\end{array}$ & , sig. 0.0 & & & & $\begin{array}{l}\text { Chi }^{2}: 544 \\
V=0.17\end{array}$ & ig. ( & $\begin{array}{l}7.51 \\
00\end{array}$ & \\
\hline
\end{tabular}

En tercer lugar, el efecto de la presencia de instancias de /d/ en la misma palabra parece ser cualitativamente distinto, puesto que no importa cómo se realiza la /d/ sino dónde se encuentra esta: delante o detrás de la variable (Tabla 14). La existencia -al margen de cómo se realice- de una /d/ siguiente 
en la misma palabra (poDido, reDada) retiene la elisión del segmento, en tanto que la /d/ previa (pediDo) favorece la elisión, incluso por encima de la frecuencia de elisión en palabras con una sola $/ \mathrm{d} /$. La estructura de la palabra parece operar al margen de la realización efectiva de /d/ previa o siguiente: da la impresión de que el hablante tiene una imagen de la forma léxica (podido, redada) y sobre ella actúa la tendencia a elidir la que sigue y a mantener la previa [po'ðio, re'ða]. No obstante, conviene tener en cuenta la interacción con el efecto del esquema acentual postónico característico de las terminaciones más frecuentes (-ado, -ada, etc.), puesto que la mayor parte de las palabras con más de una /d/ pertenece a esta serie. De hecho, el efecto de esta variable no resultó seleccionado como significativo en la predicción de la elisión de /d/ en los análisis multivariantes (Sección 3.3).

Tabla 14. Efecto de priming en la palabra sobre la elisión de /d/

\begin{tabular}{|c|c|c|c|c|c|c|c|c|}
\hline & \multicolumn{4}{|c|}{ Málaga } & \multicolumn{4}{|c|}{ Granada } \\
\hline & No ocurr & /d/ previa & /d/ sig. & Total & No ocurr. & /d/ previa & /d/ sig. & Total \\
\hline$d$ & 4225 & 314 & 919 & 5458 & 3131 & 102 & 166 & 3399 \\
\hline & 66.7 & 50.0 & 72.8 & 66.4 & 62.4 & 56.7 & 93.8 & 63.3 \\
\hline 0 & 2109 & 314 & 344 & 2767 & 1883 & 78 & 11 & 1972 \\
\hline & 33.3 & 50.0 & 27.2 & 33.6 & 37.6 & 43.3 & 6.2 & 36.7 \\
\hline Total & 6334 & 628 & 1236 & 8225 & 5014 & 180 & 177 & 5371 \\
\hline $\begin{array}{l}\mathrm{Chi}^{2}: \\
\mathrm{V}=0\end{array}$ & $\begin{array}{l}8225,2= \\
110, \text { sig. }\end{array}$ & $\begin{array}{l}8.825, \\
.000\end{array}$ & 00 & & $\begin{array}{l}\text { Chi }^{2}: 5371 \\
V=0.119\end{array}$ & $\begin{array}{c}2=75.78 \\
\text { sig. } 0.000\end{array}$ & sig. & \\
\hline
\end{tabular}

\subsubsection{Marcación léxica}

La marcación de las formas léxicas se refiere aquí a la distinción entre el léxico general y el más restringido de los nombres propios (antropónimos, topónimos) o los términos (terminologías técnicas, tecnológicas, científicas, etc.). El tratamiento desigual es el esperado entre la /d/ de deDo, debiDo y la /d/ de legraDo, MachaDo, MoneDero, etc. (Tabla 15).

Tabla 15. Efecto de la marcación léxica en la elisión de /d/

\begin{tabular}{ccccccc} 
& \multicolumn{4}{c}{ Málaga } & \multicolumn{4}{c}{ Granada } \\
\hline & Marcado & $\begin{array}{c}\text { No } \\
\text { marcado }\end{array}$ & Total & Marcado & $\begin{array}{c}\text { No } \\
\text { marcado }\end{array}$ & Total \\
d & 388 & 5077 & 5465 & 325 & 3141 & 3466 \\
& 94.9 & 64.9 & 66.4 & 82.5 & 62.1 & 63.5
\end{tabular}




\begin{tabular}{lcccccc}
0 & 21 & 2748 & 2769 & 69 & 1920 & 1989 \\
& 5.1 & 35.1 & 33.6 & 17.5 & 37.9 & 36.5 \\
Total & 409 & 7825 & 8234 & 394 & 5061 & 5455 \\
\hline Chi $^{2}: 8234,1=156.559$, sig. 0.000 & \multicolumn{2}{l}{ Chi $^{2}: 5455,1=65.821$, sig. 0.000} \\
Phi $=0.138$, sig. 0.000 & & Phi $=-0.110$, sig. 0.000
\end{tabular}

\subsubsection{Variables de hablante}

La elisión de /d/ es un proceso de variación patrocinado hoy en Andalucía por los hablantes de los grupos educacionales más bajos (especialmente los más jóvenes) contra el que reaccionan (o que no siguen) los hablantes mayores de los grupos educacionales más altos. Esto quiere decir que estamos ante un patrón de estratificación educacional jerárquico y ante una gradación de edad inversa. El papel de las diferencias de sexo no es, en general, significativo pero sí supone contrastes en los grupos educacionales y generacionales.

\subsubsection{Sexo}

Si nos fijamos en los estudios previos, las diferencias genéricas en la variación de/d/van en todas las direcciones posibles: comunidades en las que no son significativas (Valencia, Granada); comunidades en las que apuntan tendencias vernaculares masculinas (Jaén, Málaga, Las Palmas, Santa Cruz de Tenerife); comunidades que sugieren tendencias femeninas (San Juan, Santiago de los Caballeros, Castellón, Panamá). La posible relación entre estos resultados y el grado de generalización del proceso queda desmentida por los resultados de Málaga y Granada, que no son uniformes: en Málaga las diferencias, aunque débiles, existen y la tendencia es masculina $(\mathrm{H}=37.7$; $\mathrm{M}=29.5$, sig. 0.000; Phi $=-0.087$, sig. 0.000); en Granada la tendencia es femenina y las diferencias son solo aparentes $(H=36.1 ; M=36.8$; no sig. $)$.

\subsubsection{Edad}

Los estudios realizados en varias comunidades centroseptentrionales de España y en otras de América han llevado a sugerir la existencia de un proceso de restitución de /d/ por parte de los hablantes más jóvenes (Getafe, Toledo, Alcalá de Henares, Santiago de los Caballeros). Esta es una de las tendencias más claras en el español de Andalucía: tal proceso reactivo -si existe- no está patrocinado por los jóvenes, sino por los hablantes de edad intermedia y, sobre todo, los mayores. La gradación de edad (Gráfico 5) es similar en las dos ciudades andaluzas estudiadas aquí; las diferencias entre los grupos de edad son significativas (Granada, $\mathrm{Chi}^{2}=42.197(2)$, sig. 0.000, $\mathrm{V}=0.088$, sig. 0.000; Málaga, $\mathrm{Chi}^{2}=47.274(2)$, sig. 0.000, $\mathrm{V}=0.076$, sig. 
0.000) pero débiles, de modo que, como pasa en Málaga, las diferencias entre los grupos de edad intermedio y mayor son aparentes cuando se estudian con mayor detenimiento.

Gráfico 5. Gradación de edad de la elisión de /d/

Elisión de /d/

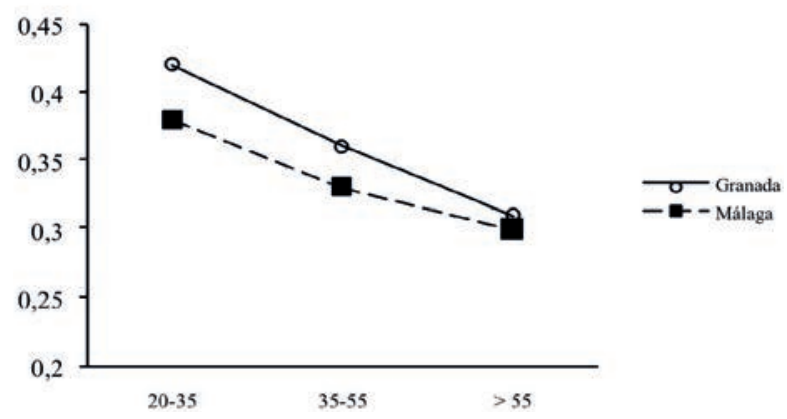

La cuestión ahora es si son los o las jóvenes los que patrocinan la elisión y cuál es el papel de las diferencias de sexo en la retracción de los maduros y los mayores (Gráfico 6). En Granada, la tendencia a la elisión es masculina y significativa $(p=0.001)$ entre los jóvenes pero se invierte en el grupo de edad intermedio en el que la elisión es una tendencia claramente femenina ( $\mathrm{p}$ $=0.000)$; en el grupo de los mayores las diferencias no existen $(\mathrm{p}=0.318)$. Las diferencias de edad son significativas en ambos sexos $(p=0.000)$.

Gráfico 6. Efecto del sexo y la edad en la elisión de /d/ en Granada

Elisión de /d/ en Granada

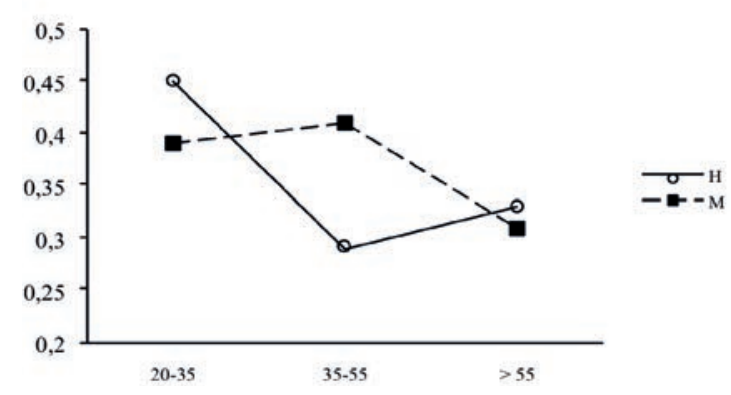

En Málaga las diferencias de edad solo existen entre las mujeres $(\mathrm{p}=$ 0.000); los hombres de las diferentes generaciones tienen comportamientos similares (Gráfico 7). Las diferencias de sexo, como refleja el gráfico, son 
significativas en los grupos de hablantes $>34$ años $(p=0.000)$, pero no entre los jóvenes $(\mathrm{p}=0.162)$.

Gráfico 7. Efecto de la edad y el sexo en la elisión de /d/ en Málaga

Elisión de /d/ en Málaga

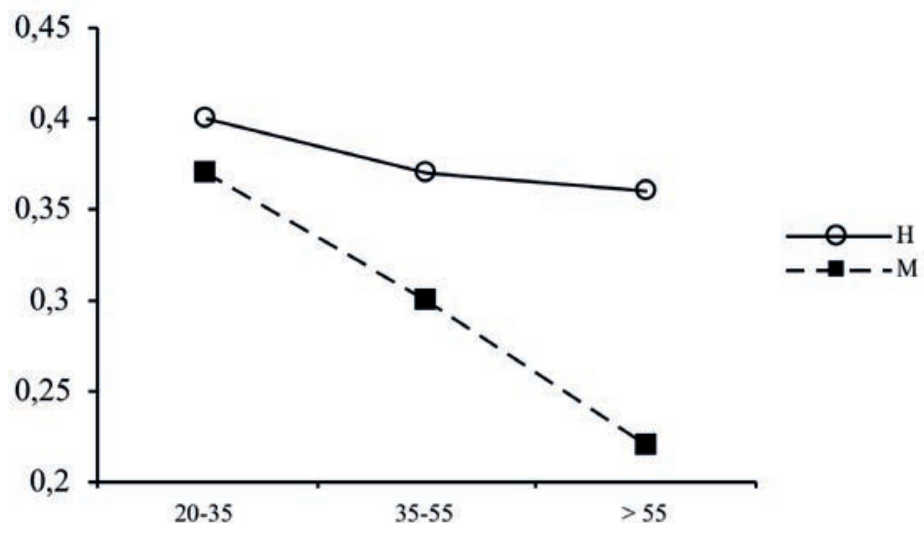

Como se observa, en Málaga la reacción de la III. a generación frente a la elisión es fundamentalmente femenina en la medida en que esta se identifica, probablemente, con un marcador masculino.

Así pues, el patrón etario aparentemente jerárquico en ambas ciudades esconde patrones cruzados de sexo y edad bien diferentes: en Granada, la reacción más fuerte es masculina de la II generación (patrón en V), en tanto que las mujeres del mismo grupo de edad lideran ahora la elisión (patrón circunflejo). Las mujeres nacidas entre 1945 y 1970 parecen haber adoptado la pronunciación de los hombres jóvenes de la generación posterior (nacidos después de 1970), en tanto que los hombres reaccionan contra ella. Es posible que dicha adopción se esté moderando actualmente. En Málaga se observa que el patrón general es producto de la fuerte reacción femenina puesto que no hay diferencias masculinas.

\subsubsection{Educación}

El patrón jerárquico es similar en Granada y Málaga, si bien llama la atención la distancia entre los hablantes con estudios secundarios y universitarios en Granada, que no existe en Málaga debido a la reacción de los hablantes de secundaria (Tabla 16). 
Tabla 16. Estratificación educacional de la elisión de /d/

Málaga

Granada

\begin{tabular}{|c|c|c|c|c|c|c|c|c|}
\hline & Primar. & Secund. & Univers. & Total & Primar. & Secund. & Univers. & Total \\
\hline Elisión & 1227 & 929 & 613 & 2769 & 833 & 683 & 473 & 1989 \\
\hline$\%$ & 52.3 & 26.8 & 25.3 & 33.6 & 50.9 & 38.6 & 23.1 & 36.5 \\
\hline Total & 2346 & 3464 & 2424 & 8234 & 1636 & 1770 & 2049 & 5455 \\
\hline $\mathrm{V}=0.2$ & ig. 0 . & 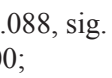 & & & $\mathrm{V}=0.23$ & 38 , sig. 0. & 000 & \\
\hline
\end{tabular}

La cuestión es saber si las diferencias de sexo desempeñan aquí algún papel importante (Tabla 17). Por un lado, en Granada estas son significativas en los dos grupos educacionales más bajos pero no en el universitario, lo que denota el cumplimiento del principio empírico de la disolución educacional del sexo (Villena y Ávila 1999). En Málaga ocurre a la inversa: no son significativas las diferencias de sexo entre los hablantes de primaria. Por otro lado, las diferencias educacionales en los grupos genéricos son significativas en ambas ciudades (Gráfico 8).

Tabla 17. Diferencias de sexo y educación en la elisión de /d/

\begin{tabular}{cccccccccccc}
\multicolumn{1}{c}{ Granada } & \multicolumn{1}{c}{ Málaga } \\
\hline & Prim. & Sec. & Univ. & Total & $\mathrm{Chi}^{2}$. & Prim. & Sec. & Univ. & Total & $\mathrm{Chi}^{2}$ \\
& & & & & & & & & & \\
$\mathrm{H}$ & 335 & 352 & 195 & 882 & .000 & 668 & 581 & 322 & 1571 & .000 \\
& 46.4 & 41.4 & 22.3 & & & 50.6 & 33.3 & 29.2 & & \\
Total & 722 & 851 & 873 & 2446 & & 1321 & 1747 & 1103 & 4171 & \\
M & 498 & 331 & 278 & 1107 & .000 & 559 & 348 & 291 & 1198 & .000 \\
& 54.5 & 36.0 & 23.6 & & & 54.5 & 20.3 & 22.0 & & \\
Total & 914 & 919 & 1176 & 3009 & & 1025 & 1717 & 1321 & 4063 & \\
Sig. & .001 & .021 & .489 & & & .056 & .000 & .000 & & \\
\hline
\end{tabular}

Lo más interesante es la tendencia femenina a la elisión entre los hablantes menos instruidos que invierte lo que ocurre en los demás grupos educacionales. 
Gráfico 8. Diferencias de sexo en la estratificación educacional de la elisión de /d/

Elisión de /d/ en Granada

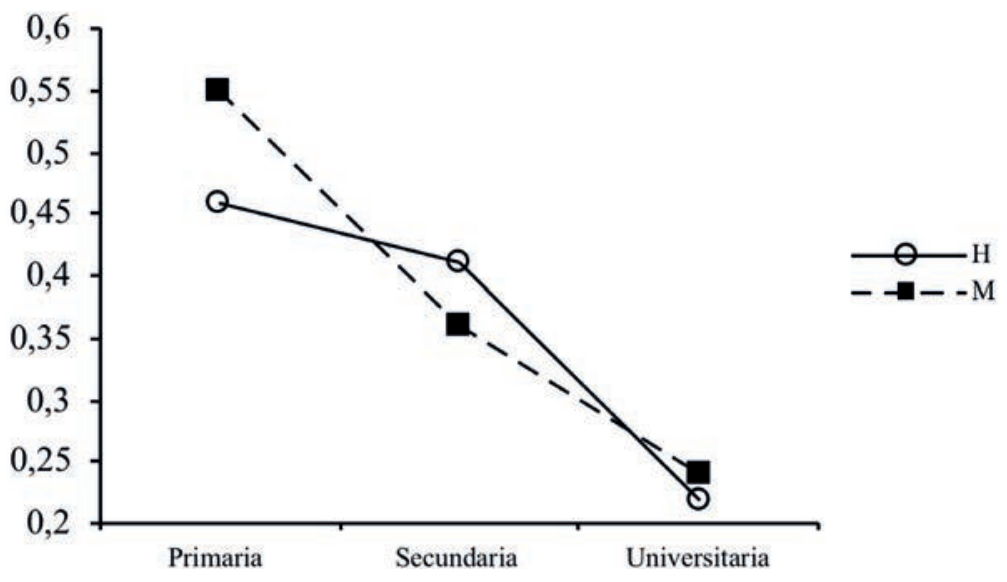

Elisión de /d/ en Málaga

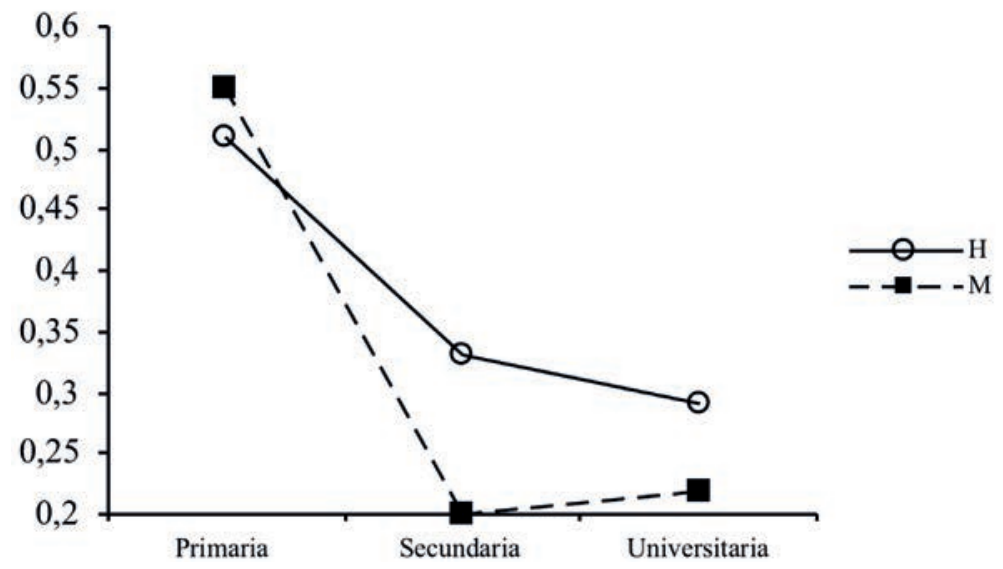

\subsubsection{Relexificación}

Como suponíamos, la retracción de la elisión relacionada con la gradación de edad (3.2.2.2) supone, en cierto modo, un proceso relexificador y está liderada por los hablantes más altos en la estratificación educacional de todas las edades. Sin embargo, la reacción es más fuerte entre los hablantes 
mayores de 35 años y, especialmente los que han superado los 55 años (Tabla 18 y Gráfico 9).

En Málaga destacan dos hechos: en primer lugar, no son los jóvenes (I) de nivel educacional bajo, sino los mayores de 35 años los que lideran la elisión, sin que se perciban diferencias entre los grupos de edad II y III; en segundo lugar, la reacción de los hablantes con estudios secundarios y universitarios afecta a todas las edades pero sigue el patrón inverso esperado y comentado hasta aquí.

En Granada destacan asimismo dos cuestiones: en primer lugar, la elisión la lideran los jóvenes sin estudios; en segundo lugar, los hablantes de instrucción secundaria y universitaria reaccionan contra la elisión con un patrón débil que resulta alterado por la fuerte inflexión negativa de los universitarios de la III generación.

Las diferencias son significativas en Málaga a través de los grupos de edad y de los grupos educacionales. En Granada, sin embargo, no existen diferencias de edad entre los hablantes de secundaria.

Tabla 18. Efecto de la interacción de la edad y la educación en la elisión de /d/

$$
\text { Málaga Granada }
$$

\begin{tabular}{ccccccccccc} 
& Prim. & Sec. & Univ. & Total & Sig. & Primar. & Sec. & Univ. & Total & Sig. \\
\hline I & 318 & 393 & 293 & 1004 & .000 & 311 & 253 & 195 & 759 & .000 \\
& 47.7 & 36.0 & 34.3 & 38.4 & & 56.2 & 41.7 & 30.1 & 42.0 & \\
Total & 667 & 1092 & 854 & 2613 & & 553 & 607 & 647 & 1807 & \\
II & 472 & 289 & 176 & 937 & .000 & 214 & 236 & 197 & 647 & .000 \\
& 53.5 & 27.5 & 19.7 & 33.2 & & 47.2 & 37.8 & 26.9 & 35.7 & \\
\multirow{2}{*}{ Total } & 883 & 1050 & 893 & 2826 & & 453 & 624 & 733 & 1810 & \\
III & 437 & 247 & 144 & 828 & .000 & 308 & 194 & 81 & 583 & .000 \\
& 54.9 & 18.7 & 21.3 & 29.6 & & 48.9 & 36.0 & 12.1 & 31.7 & \\
\multirow{2}{*}{ Total } & 769 & 1322 & 677 & 2795 & & 630 & 539 & 669 & 1838 & \\
& .015 & .000 & .000 & & & .008 & .126 & .000 & & \\
\hline
\end{tabular}

En resumen, la reacción conservadora frente al rasgo no marcado de la elisión se manifiesta en Málaga como una frontera entre los hablantes con o sin estudios secundarios y universitarios y entre menores y mayores de 35 años. Lo mismo ocurre en Granada, pero esa frontera no es tan nítida puesto que los hablantes de la II generación no reaccionan siguiendo un patrón inverso tan claro como en Málaga; además, la reacción de los universitarios mayores de 55 años es muy fuerte. 
Gráfico 9. Comparación del efecto de la edad y la educación en la elisión de /d/

\section{Elisión de /d/ en Málaga}

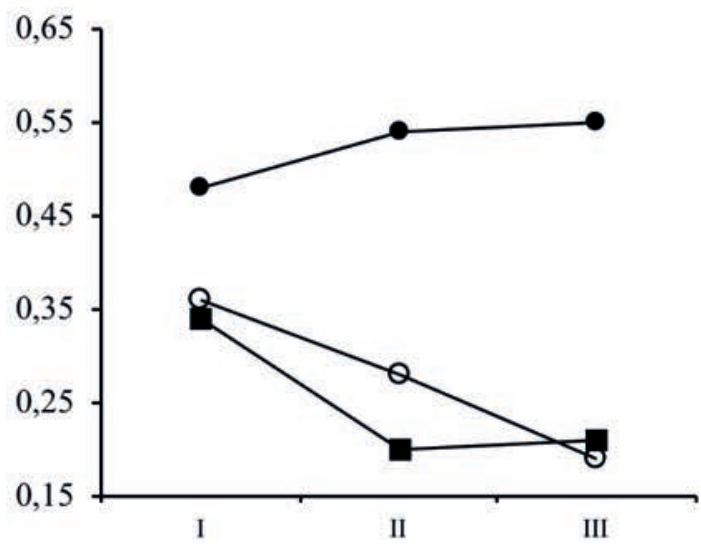

Elisión de /d/ en Granada

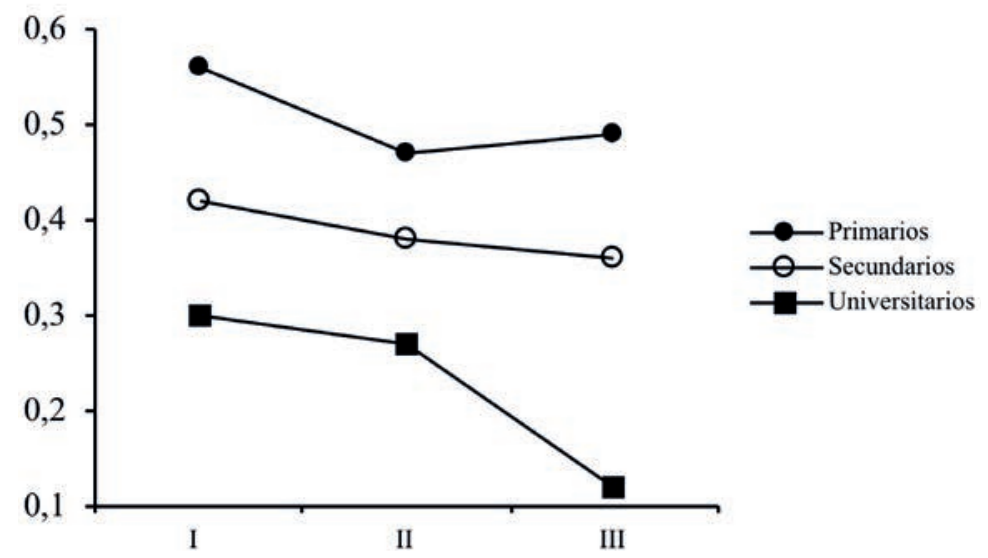

3.2.2.5. El efecto iluminador del sexo

Cuando consideramos las diferencias de sexo en el esquema anterior en las dos ciudades descubrimos que la reacción universitaria constatada en Granada es fundamentalmente masculina y que la aparente estratificación débil esconde una fuerte reacción también masculina de la generación intermedia. En Málaga el efecto del sexo se manifiesta en términos de frecuencia únicamente puesto que los patrones son similares: segregación 
estratificacional clara entre los niveles educacionales bajos y el resto (Tablas 19 y 20; Gráfico 10).

Tabla 19. Efecto de la interacción de la edad y la educación en la elisión de /d/ entre hablantes masculinos en Málaga y Granada

Málaga Granada

Prim. Sec. Univ. Total Chi2 Prim. Sec. Univ. Total Sig.

\begin{tabular}{|c|c|c|c|c|c|c|c|c|c|c|}
\hline \multirow[t]{2}{*}{1} & 159 & 282 & 138 & 579 & sig. .004 & 149 & 145 & 106 & 400 & sig. .000 \\
\hline & 46.5 & 39.1 & 34.7 & 39.6 & $\mathrm{~V}=.086$ & 55.0 & 47.0 & 34.0 & 45.0 & $\mathrm{~V}=.174$ \\
\hline Total & 342 & 722 & 398 & 1462 & & 271 & 306 & 312 & 889 & \\
\hline \multirow[t]{2}{*}{ II } & 193 & 156 & 109 & 458 & sig. .000 & 63 & 90 & 59 & 212 & sig. .002 \\
\hline & 47.8 & 36.0 & 27.9 & 37.3 & $V=.166$ & 34.4 & 32.7 & 21.1 & 28.8 & $\mathrm{~V}=.132$ \\
\hline Total & 404 & 433 & 390 & 1227 & & 183 & 275 & 279 & 737 & \\
\hline \multirow[t]{2}{*}{ III } & 316 & 143 & 75 & 534 & sig. .000 & 123 & 117 & 30 & 270 & sig .000 \\
\hline & 55.0 & 24.2 & 23.8 & 36.0 & $\mathrm{~V}=.314$ & 45.9 & 43.3 & 10.6 & 32.9 & $\mathrm{~V}=.344$ \\
\hline Total & 575 & 592 & 315 & 1482 & & 268 & 270 & 282 & 820 & \\
\hline Chi2 & .0019 & .000 & .005 & .130 & & .000 & .001 & .000 & .000 & \\
\hline V & .078 & .141 & .098 & .031 & & .161 & .126 & .232 & .145 & \\
\hline
\end{tabular}

$\mathrm{Chi}^{2}$ total, educación $=$ sig. .000

$\mathrm{V}=.184$

$\mathrm{Chi}^{2}$ total, educación $=$ sig. .000

$\mathrm{V}=.217$

Tabla 20. Efecto de la interacción de la edad y la educación en la elisión de /d/ entre hablantes femeninos en Málaga y Granada

Málaga Granada

Prim. Sec. Univ. Total Chi2 Prim. Sec. Univ. Total Chi2

\begin{tabular}{lcccccccccc}
\hline I & 159 & 111 & 155 & 425 & sig. .000 & 161 & 108 & 89 & 359 & sig. .000 \\
& 48.9 & 30.0 & 34.0 & 36.9 & $\mathrm{~V}=.160$ & 57.4 & 35.9 & 26.6 & 39.1 & $\mathrm{~V}=.263$ \\
Total & 325 & 370 & 456 & 1151 & & 282 & 301 & 335 & 918 & \\
\hline II & 279 & 133 & 67 & 479 & sig. .000 & 151 & 146 & 138 & 435 & sig. .000 \\
& 58.2 & 21.6 & 13.3 & 30.0 & $\mathrm{~V}=.411$ & 55.9 & 41.8 & 30.4 & 40.5 & $\mathrm{~V}=.207$ \\
\multirow{2}{*}{ Total } & 479 & 617 & 503 & 1599 & & 270 & 349 & 454 & 1073 & \\
\hline III & 121 & 104 & 69 & 294 & sig. .000 & 185 & 77 & 51 & 313 & sig. .000 \\
& 54.8 & 14.2 & 19.1 & 22.4 & $\mathrm{~V}=.353$ & 51.1 & 28.6 & 13.2 & 30.7 & $\mathrm{~V}=.353$ \\
Total & 221 & 730 & 362 & 1313 & & 362 & 269 & 387 & 1018 &
\end{tabular}




$\begin{array}{lllll}\text { Chi2 } & .033 & .000 & .000 & .000 \\ \mathrm{~V} & .081 & .150 & .217 & .124\end{array}$

Chi2 total, educación $=$ sig. 0.000 $\mathrm{V}=.320$

$\begin{array}{llll}.236 & .003 & .000 & .000 \\ .056 & .112 & .176 & .090\end{array}$

Chi2 total, educación $=$ sig. 0.000

$\mathrm{V}=.265$

Gráfico 10. Efecto de la interacción de la edad, la educación y el sexo en la elisión de /d/ en Málaga y Granada

Granada

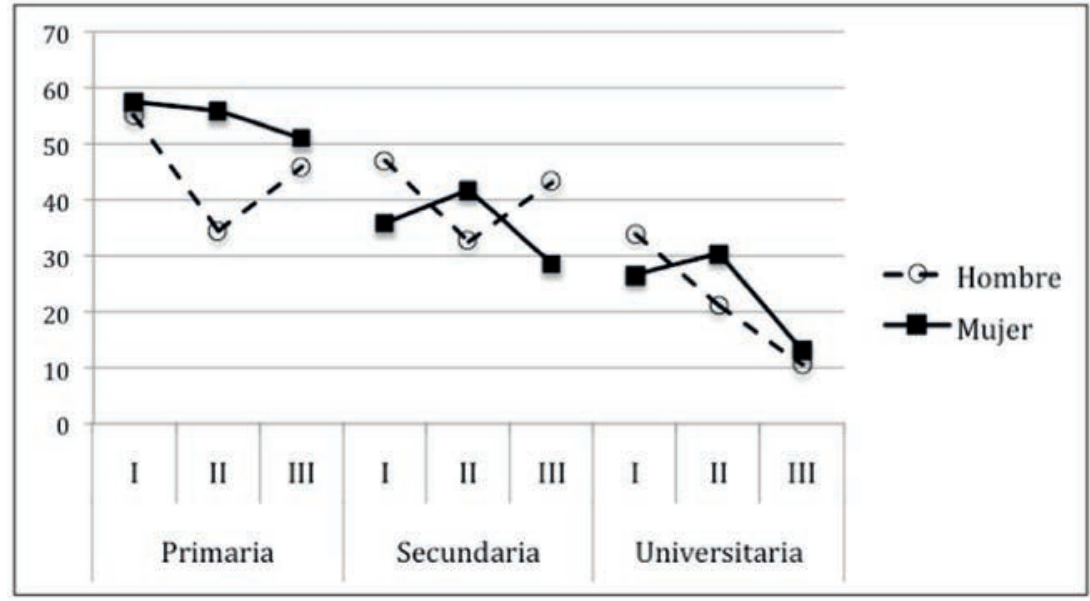

Málaga

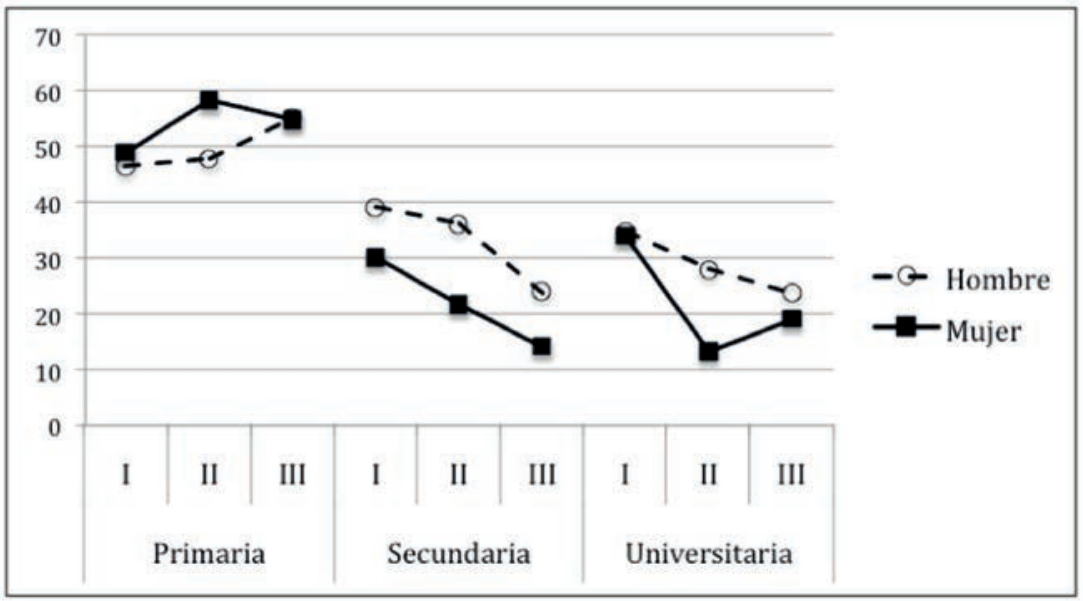


Así pues, existe una reacción constante que aumenta con el nivel educacional en ambos sexos y ambas ciudades. Esta reacción «desde arriba» aumenta conforme aumenta la edad con una excepción: la reacción de los hablantes masculinos de la generación intermedia en Granada; tanto los de nivel primario como secundario hacen descender dramáticamente la frecuencia de elisión aproximándola a la de los jóvenes universitarios, en contraste con las mujeres.

\subsection{ANÁLISIS MULTIVARIANTE COMPARATIVO}

\subsubsection{Análisis comparativo}

En este trabajo, el análisis multivariante de /d/ intervocálica en Granada y Málaga va más allá de la construcción de modelos estadísticos paralelos de esta variable fonológica. Es verdad que necesitamos considerar separadamente qué variables -de las analizadas hasta aquí siguiendo procedimientos bivariantes-resultan significativas cuando se incluyen todas en un modelo probabilístico, cuál es la jerarquía de sus correspondientes efectos sobre la elisión de /d/ y qué variantes tienen efecto favorecedor y cuáles restrictivo. Sin embargo, como el objetivo consiste en saber qué relaciones se dan entre los corpus de ambas variedades urbanas y en interpretar sus similaridades y diferencias intra e interdialectales, se hace necesario contrastar los patrones de variación de modo que sea posible captar ámbitos de divergencia y separación incipiente entre patrones comunes y, a la inversa, indicios de convergencia y aproximación entre patrones diferentes.

Hasta aquí se ha podido comprobar la estrecha semejanza entre los patrones sociolingüísticos de las dos variedades urbanas y se ha apuntado la existencia de incipientes diferencias cuantitativas entre ellas en algunas formas o bajo determinadas condiciones. Estas formas o condiciones constituyen un ámbito de conflicto (conflict site), que es una noción clave en la perspectiva sociolingüística comparativa (Tagliamonte 2012: 162-176) y que se define como "[...] a form or class of forms that differs functionally and/or structurally and/or quantitatively accross the varieties in question" (ibíd., 164). Al analizar cuantitativamente los patrones de distribución en ámbitos gramaticales en los que las variedades son consideradas diferentes, puede precisarse la naturaleza exacta de las similitudes entre los conjuntos de datos y, a la inversa, cuando las variedades son muy próximas, es posible captar con detalle las diferencias incipientes entre ellas.

En el análisis se cumplen los requisitos para la comparación (Tagliamonte 2012: 166-167; Meyerhoff 2009; Poplack y Tagliamonte 2001) y los 
resultados se comparan mediante el contraste de los grupos de factores en ambos modelos estadísticos en función de tres líneas de evidencia: 1) significación estadística (grupos de factores significativos en los dos análisis); 2) peso relativo (jerarquía entre los grupos de factores) y 3) jerarquía de condicionamientos (orden de los factores dentro de cada grupo de factores). Los resultados muestran que ambas comunidades funcionan como una sola; la diferencia consiste en que una de ellas (Granada) va algo por delante de la otra (Málaga): el avance del cambio entre los hablantes masculinos más jóvenes de estatus bajo en Málaga se amplía a los hablantes de ambos sexos de menos de 55 años y de educación media o primaria en Granada. Esto quiere decir que la elisión solo es marcador de género en Málaga y que la polarización etaria (jóvenes frente al resto) y educacional (hablantes con educación obligatoria frente al resto) que se mantiene en Málaga, y que probablemente existió en Granada tiempos atrás, se ha diluido en una gradación suave de edad y en una estratificación educacional débil (Tabla 21, p. 316).

En la Tabla 21 se muestran los resultados del análisis comparativo de regresión logística, que se ajusta perfectamente a la estructura de los datos que se han tratado aquí (variables independientes nominales y variable dependiente nominal con dos valores). Los grupos de factores seleccionados en el análisis fueron los siguientes:

\begin{tabular}{lll}
\hline Variable & Variantes & Ejemplo \\
\hline 1 CatG*Term & Ado & hablaDo, soldaDo \\
& Sufijos y asimil. & $\begin{array}{l}\text { saliDo, toDo, nada } \\
\text { caDera }\end{array}$ \\
\hline 2 Educación & Obligatoria & \\
& Secundaria & \\
& Universitaria & \\
\hline 3 Edad & I. $^{\text {a }}$ Gen. & \\
& II. ${ }^{a}$ Gen. & \\
& III. ${ }^{a}$ Gen & \\
\hline Tipo discursivo & Diálogo & \\
& Resto & \\
\hline 5 Sexo & Hombre & \\
& Mujer & \\
\hline
\end{tabular}


De estos grupos merece especial comentario el primero, que es un grupo interactivo de entorno fónico y categoría gramatical. Los mejores resultados se producen cuando se oponen los casos de sufijos o terminaciones en /ado/ de cualquier categoría gramatical a, por un lado, los casos de otros sufijos o terminaciones en /ido, ida, ada/ y a los pronombres asimilados a estos en /odo, oda, ada/ y, por otro lado, a todos los demás casos que incluyen cualquier combinación de vocales. Esta recodificación se basa, en realidad, en la frecuencia de ocurrencia de los tipos en el corpus estudiado (1.3 y 1.4).

En análisis complementarios previos se rechazó la existencia de multicolinealidad o interacción, particularmente entre los hablantes y las variables sociales (Johnson 2008: 363-365; Tagliamonte 2012: 120-161), de modo que se presentan los resultados del análisis bajo el formato clásico Varbrul. No se ha tenido en cuenta, sin embargo, el posible efecto de la variación léxica sobre las variables internas. El ajuste de ambos modelos a los datos es bueno, tal y como indica el logaritmo de verosimilitud, en el que se basa el análisis $(\mathrm{p}=0.0000)$; por su parte, los grupos de factores (GF) significativos son los mismos en los dos análisis, con la salvedad ya comentada del sexo, que es significativo en Málaga pero no en Granada y con la misma jerarquía: $10^{\circ}$ grupo interactivo de categoría gramatical y terminación; $2 .^{\circ}$ educación; $3 .^{\circ}$ edad; $4 .^{\circ}$ tipo discursivo.

En la jerarquía interna de cada GF se localizan los contrastes: en primer lugar, en la variable gramatical/fonológica (Cat. gram y term.) se confirma la verosimilitud de la hipótesis inicial sobre el efecto decisivo de la frecuencia tipo: la elisión depende en las dos comunidades de la terminación (frente a la raíz) más frecuente; es decir, ante todo, del sufijo -ado, sin que la categoría gramatical tenga nada que ver. Los hablantes reconocen los sufijos (ado > ido > a ada) e, incluso, extienden esta estructura frecuente a formas lexemáticas también muy frecuentes (todo, toda, nada). El peso probabilístico de la terminación en -ado (.91 en Granada y .94 en Málaga) sobre la elisión es abrumador, de tal modo que el factor formado por los sufijos y asimilados tiene muy pequeño efecto positivo (.52 en Málaga) o está en el límite (.46 en Granada). Por supuesto, los lexemas desfavorecen claramente la elisión por diversas razones ya expuestas (Sección 1).

En lo que se refiere a las variables de hablante, el contraste se manifiesta en el mismo sentido en los tres grupos de factores: educación, edad y sexo. La estructura de estratificación educacional débil, gradación suave de edad y disolución del contraste genérico en Granada pone de manifiesto el efecto favorecedor de la elisión por parte de los grupos educacionales y de edad intermedios; en Málaga, por el contrario, la desfavorecen de modo que se produce estratificación educacional y diferencias de edad polarizadas. El hecho de que el sexo no fuese seleccionado como grupo significativo en el 
análisis del conjunto de datos de Granada indica que el contraste genérico, que existe débilmente aún en Málaga, ha dejado de tener efecto en la elisión de /d/ en Granada.

El resultado del análisis comparativo de los corpus de las dos comunidades indica pues que en ambas ciudades funcionan los mismos factores y que las diferencias de comunidad de habla interaccionan en alguna medida con la educación, la edad y el sexo.

Tabla 21. Regresión logística de los factores internos y externos sobre la probabilidad de elisión de /d/ en Granada y Málaga. Análisis comparativo

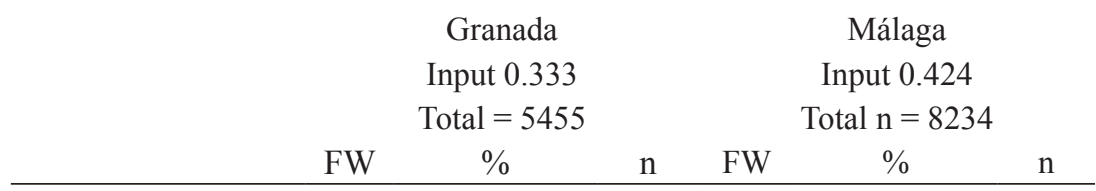

Condicionamientos

Cat. gram y term.

-ado

Sufijos y asimil.

Lexemas

\begin{tabular}{|l|}
.91 \\
\hline .46 \\
\hline .10
\end{tabular}

86

$999 \quad .94$

86

1597

Rango

81

$2415 \quad .52$

$2041 \quad .06$

35

3658

8

4

2979

Educación

Obligatoria

Secundaria

Universitaria

.68

.51

Rango

\begin{tabular}{l|l|}
1636 & .68 \\
2049 & \\
1770 & .33 \\
\cline { 2 - 2 } & 35
\end{tabular}

52

2346

$26 \quad 5888$

Edad

I. ${ }^{\mathrm{a}} \mathrm{Gen}$.

II. ${ }^{\mathrm{a}}$ Gen.

III. ${ }^{a}$ Gen.

.60

42

\begin{tabular}{l|l|}
1807 & .58 \\
1810 & .42 \\
1838 & .42 \\
\cline { 2 - 2 } & 16
\end{tabular}

38

5621

Rango

36

32

21

31

2613

Tipo discursivo

$\begin{array}{lllcccc}\text { Diálogo } & .60 & 38 & 5018 & .55 & 43 & 3249 \\ \text { Resto } & .40 & 23 & 435 & .45 & 28 & 4985 \\ \text { Rango } & 20 & & & 10 & & \end{array}$


Sexo

Hombre

Mujer

\begin{tabular}{|c|}
\hline .54 \\
.46 \\
\hline 8
\end{tabular}

38

4071

Rango

30

4063

Convergencia en la iteración 7

Convergencia en la iteración 7

Log likelihood $=-2390.706$ Sig. $=0.021$

Máx. likelihood posible $=-2292.864$

Log likelihood $=-3156.572$ Sig.

$=0.000$ Máx. likelihood posible $=$

Ajuste: X-cuadrado(42) $=195.685$, rechazo, $\mathrm{p}=0.0000$

-3014.925 Ajuste: X-cuadrado(42)

$=283.294$, rechazo, $\mathrm{p}=0.0000$

\subsubsection{Análisis comparativo integrado}

La ventaja que tiene trabajar con conjuntos de datos obtenidos y operacionalizados en el marco de un proyecto de investigación común es la compatibilidad entre estos y la facilidad para llevar a cabo análisis comparativos. Los resultados del análisis comparativo anterior han puesto de manifiesto la existencia de ámbitos de conflicto entre las dos variedades, a partir de los cuales es posible dar un paso más en la simplificación del proceso analítico. En la Tabla 22 se muestran los resultados del análisis comparativo integrado de los dos conjuntos de datos. Esto quiere decir que se trataron como input del análisis los datos de ambas ciudades y se recodificaron los GF de modo que fuese posible recoger las características definidas en los ámbitos de conflicto.

Lo más notable es que -como era de esperar- el GF que separa a los hablantes por la comunidad de habla a la que pertenecen no fue seleccionado como significativo en el análisis. Tratados pues los datos como pertenecientes a la misma comunidad de habla, se confirma la misma jerarquía de GF con la inclusión del sexo del hablante como grupo significativo: el carácter fuertemente condicionado por la terminación muy frecuente en -ado, el estatus educacional bajo (enseñanza obligatoria en Málaga pero también la secundaria en Granada), la edad joven (I. ${ }^{a}$ generación en Málaga pero también la II. ${ }^{\mathrm{a}}$ generación en Granada) y el sexo (hablantes masculinos en Málaga). La diferencia con respecto al análisis previo está en el último grupo que ahora es la marcación técnica de la palabra con /d/. 
Tabla 22. Regresión logística de los factores internos y externos sobre la probabilidad de elisión de /d/ en Granada y Málaga. Análisis integrado

Condicionamientos

$$
\begin{gathered}
\text { Granada/Málaga } \\
\text { Input } 0.508 \\
\text { Total }=13689
\end{gathered}
$$

\begin{tabular}{lll} 
FW & $\%$ & $\mathrm{n}$ \\
\hline
\end{tabular}

Cat. gram y term.

-ado .89

84

2639

Sufijos y asimilados

.49

44

4223

Lexemas

.11

10

6827

Rango

78

Educación

Primaria

.67

52

3982

Secundaria Granada

.52

39

1770

Resto

.31

25

7937

Rango

36

Edad

I. ${ }^{\mathrm{a}} \mathrm{Gen}$.

.57

40

4420

II. ${ }^{a}$ Gen. Granada

.52

36

1810

Resto

.41

32

7459

Rango

16

Sexo

Hombre Málaga

.54

38

4171

Resto

.46

34

9518

Rango

8

Especialización

Marcado

.52

36

7937

No marcado

.48

26

12623

Rango

4

Convergencia en la iteración 8

Log likelihood $=-5728.647 \mathrm{Sig} .=0.004$

Máx. likelihood posible $=-5490.176$

Ajuste: $X$-cuadrado $(63)=476.942$, rechazo, $p=0.0000$ 


\section{CONCLUSIONES}

El análisis comparativo de los datos de las dos comunidades de habla pone de manifiesto que subyacen esencialmente los mismos patrones en la conducta lingüística de los hablantes de ambas ciudades. Las diferencias geográficas humanas (tipo de inmigración, condiciones de vida económica y social) y la propia evolución sociolingüística respectiva (Granada, ciudad universitaria de pasado fonológico seseante y Málaga, ciudad abierta al mar y al comercio, de pasado fonológico ceceante) no son óbice para que, vistas desde fuera y centrándonos en la variación de la /d/ intervocálica, estemos tentados de considerarlas -como en otros respectos-como una sola comunidad de habla.

Los resultados de los análisis multivariantes comparativos (separados e integrados) ponen en evidencia que por debajo del uso efectivo están los mismos patrones de variación: la elisión tiende a ser más frecuente entre los jóvenes de estatus bajo, en palabras no especializadas, cuando los hablantes dialogan, y está fuertemente condicionada por la frecuencia de inventario, que determina su casi regularización en los participios verbales y sus derivados. Las diferencias (conflict sites) indican tendencias comprobadas en el sentido de una estratificación educacional y una gradación de edad más abrupta en Málaga que en Granada.

Finalmente, si tenemos en cuenta los resultados en Andalucía, notamos un contraste notable frente a las demás áreas del español castellano: son los jóvenes los que patrocinan el cambio. Dado el largo trecho recorrido por dicho cambio erosivo, podemos estar ante una reactivación reciente (jóvenes) o ante una reacción (adultos y mayores). Si es así, Granada avanza y Málaga va más retrasada. Tal es la única diferencia entre dos ciudades hermanas.

\section{REFERENCIAS BIBLIOGRÁFICAS}

Alba, Orlando. 1999. Elisión de la /d/ intervocálica postónica en el español dominicano. En Eduardo Ferastieri et al. (eds.). Estudios de lingüistica hispánica: homenaje a María Vaquero. San Juan: Universidad de Puerto Rico, pp. 3-21.

Almeida, Manuel. 2009. Priming y efectos mecánicos en la variación lingüística: el (de) queísmo en una comunidad canaria. Boletín de Filología 44: 11-37.

2011. Restricciones sociolingüísticas en un caso de alternancia (morfo) fonológica: /d/ intervocálica en una comunidad de habla canaria. Lingüística Española Actual 33/1: 29-53.

Ávila Muñoz, Antonio. 1999. Léxico de frecuencia del español hablado en la ciudad de Málaga. Málaga: Universidad. 
Blas Arroyo, José LuIs. 2007. Competing norms in a bilingual community. Ethnolinguistic and social factors in the reversal of a change in progress in peninsular Spanish. Sociolinguistic Studies 1/3: 383-414.

Bybee, JoAn .2002. Word frequency and contexto of use in the lexical difusión of phonetically conditioned sound change. Language Variation and Change 14: 261-290.

2007. Frequency of use and the organization of language. Oxford: Oxford University Press.

Cedergren, Henrietta. 1972. Interplay of social and linguistic factors in Panama. Tesis Doctoral. Cornell University.

D’Introno, Francesco y Juan M. Sosa. 1986. Elisión de la /d/ en el español de Caracas: aspectos sociolingüísticos e implicaciones teóricas. En R. A. Núñez Cedeño et al. (eds.). Estudios sobre la fonología del español del Caribe. Caracas: La Casa de Bello, pp. 135-163.

Gómez Molina, José R. 2013. Interacción de factores internos y externos en la pronunciación de la /d/ intervocálica. En José R. Gómez Molina (ed.): El español de Valencia. Estudio sociolingüístico. Berna: Peter Lang, pp. 31-68.

Johnson, KeIth. 2008. Quantitative methods in linguistics. Malden, MA: Blackwell.

Kiparsky, Paul. 1971. Historical linguistics. En W. Dingwall (ed.). A survey of linguistic science. College Park, MD: Maryland University, pp. 577-649.

1982. Explanation in phonology. Dordrecht: Foris.

Labov, William. 1994. Principios del cambio lingüístico. Volumen I: factores internos. Madrid: Gredos, 1996.

López Morales, Humberto. 1983. Estratificación social del español de San Juan de Puerto Rico. México: UNAM.

Meyerhoff, Miriam. 2009. Replication, transfer, and calquing: Using variation as a tool in the study of language contact. Language Variation and Change 21/2, pp. 297-317.

Molina Martos, Isabel y Florentino Paredes García. 2015. La conservación de la dental -/d/- en el distrito de Salamanca. En Ana M. ${ }^{a}$ Cestero, Isabel Molina y Florentino Paredes (eds.). Patrones sociolingüísticos de Madrid. Berna: Peter Lang, pp. 63-89.

2014. Sociolingüística de la elisión de la dental /d/ en Madrid (distrito de Salamanca). Cuadernos de Lingüistica 2: 55-114.

Moya Corral, Juan Antonio. 2012. Perdido. Resultados de la terminación -ido en el corpus PRESEEA-Granada. RFULL 30: 113-132.

Moya Corral, Juan Antonio y Emilio García Wiedemann. 2009. La elisión de/d/ intervocálica en el español culto de Granada: factores lingüísticos. Pragmalingüística 17: 93-123.

Moya Corral, Juan Antonio, Emilio García Wiedemann, Esteban Montoro, Marcin Sosinsky, M. ${ }^{a}$ Concepción Torres, Francisca Pose y Elisabeth Melguizo. 2012. La/d/ intervocálica en Granada: factores lingüísticos y sociales. Español hablado. Estudios sobre el corpus PRESEEA-Granada. Varsovia: Instituto de Estudios Ibéericos e Iberoamericanos, pp. 95-148.

Naro, Anthony. 1981. The social and structural dimensions of a syntactic change. Language 57/1: 63-98.

Pierrehumbert, Janet B. 2001. Exemplar dynamics: Word frequency, lenition and contrast. En J. Bybee y P. Hooper (eds.). Frequency effecs and emergent grammar. Amsterdam: John Benjamins, pp. 137-157.

Poplack, Shana. 1979. Function and process in a variable phonology. Tesis Doctoral. Filadelfia: University of Pennsylvania.

Poplack, Shana y Sali Tagliamonte. 2001. African American English in the Diaspora: Tense and aspect. Malden: Blackwell. 
Samper Padilla, José Antonio. 2011. Elisión de /d/ en los niveles de estudios primario y universitario de Las Palmas de Gran Canaria. Comparación con otras comunidades de habla. En José J. de Bustos Tovar et al. (eds.). Sintaxis y análisis del discurso hablado en español. Homenaje a Antonio Narbona, vol. I. Sevilla: Universidad, pp. 409-424.

Scherre, Maria M. y Anthony J. Naro. 1991. Marking in discourse. 'Birds of a feather'. Language Variation and Change 3: 23-32.

1997. A concordância de número no português do Brasil: um caso típico de variação inerente. En D. da Hora (ed.). Diversidade lingüística no Brasil. João Pessoa: Idéia, pp. 93-114.

Tagliamonte, Sali A. 2012. Variationist sociolinguistics. Change, observation, interpretation. Oxford: Wiley-Blackwell.

Tejada Giráldez, María de la Sierra. 2015. Convergencia y divergencia entre comunidades de habla: a propósito de la /s/ implosiva. Contribución al estudio de los patrones sociolingüísticos del español de Granada. Tesis doctoral. Granada: Universidad.

Vida Castro, Matilde. 2004. Estudio sociofonológico del español hablado en la ciudad de Málaga. Condicionamientos sobre la variació de /-s/ en la distensión silábica. Alicante: Universidad.

Villena Ponsoda, Juan Andrés. 2012. Estatus, red e individuo. Fundamentos del análisis escalonado de la variación lingüística. Elisión de /d/ en el español de Málaga. En José F. Val Álvaro et al. (eds.). De la unidad del lenguaje a la diversidad de las lenguas. Actas del X Congreso de Lingüistica General de Zaragoza. Zaragoza: Universidad, pp. 953-970.

Villena Ponsoda, Juan Andrés y Antonio M. Ávila Muñoz. 1999. La disolución cultural del sexo. En M. ${ }^{a}$ Dolores Fernández de la Torre, M. A. Medina Guerra y L. Taillefer (eds.). El sexismo en el lenguaje, vol I. Málaga: Diputación Provincial, pp. 107-142.

Villena Ponsoda, Juan Andrés, Francisco Díaz Montesinos, Antonio Ávila Muñoz y María de la Cruz Lasarte Cervantes. 2011. Interacción de factores fonéticos y gramaticales en la variación fonológica: la elisión de /d/ intervocálica en la variedad de los hablantes universitarios de la ciudad de Málaga. En Yolanda Congosto Martín y Elena Méndez García de Paredes (eds.). Variación lingüistica y contacto de lenguas en el mundo hispánico. In memoriam Manuel Alvar. Francfort/Madrid: Vervuert/Iberoamericana, pp. 311-359. 